\title{
A Digital Method of Evaluating Nonlinear Frequency Response Functions Based on Conditioned Spectral Analyses
}

\section{Wen-Tao Liu}

University of Jinan https://orcid.org/0000-0001-9627-0168

Yun Zhang ( $\nabla$ zhangyun@mail.tsinghua.edu.cn )

Tsinghua University

Shou-Ren Wang

University of Jinan

\section{Original Article}

Keywords: Nonlinear system, Frequency response function, Conditioned spectral analysis, System identification

Posted Date: January 15th, 2021

DOI: https://doi.org/10.21203/rs.3.rs-141383/v1

License: (c) (i) This work is licensed under a Creative Commons Attribution 4.0 International License. Read Full License 


\section{Title page}

\section{A Digital Method of Evaluating Nonlinear Frequency Response Functions Based on Conditioned Spectral Analyses}

Wen-Tao Liu, born in 1982, is currently a lecturer at School of Mechanical Engineering, University of Jinan, China. He received his PhD degree from Tsinghua University, China, in 2016. His research interests include diagnoses of mechanical failure and intelligent manufacturing technologies.

Tel: +86-531-82765476; E-mail: me_liuwt@ujn.edu.cn

Yun Zhang, born in 1974, is currently an associate research fellow at Beijing Key Lab of Precision/Ultra-precision Manufacturing Equipment and Control, Tsinghua University, China. He received his $\mathrm{PhD}$ degree from Tsinghua University, China, in 2004. His research interests include precision or ultra-precision machining, intelligent manufacturing and optical detective technologies.

Tel: +86-010-62773264; E-mail: zhangyun@mail.tsinghua.edu.cn

Shou-Ren Wang, born in 1966, is currently a professor at School of Mechanical Engineering, University of Jinan, China. He received his $\mathrm{PhD}$ degree from Shandong University, China, in 1996. His research interests include intelligent manufacturing processes and equipment.

Tel: +86-531-87109072; E-mail: me_liuwt@ujn.edu.cn

Corresponding author: Yun Zhang E-mail: zhangyun@mail.tsinghua.edu.cn 


\title{
A Digital Method of Evaluating Nonlinear Frequency Response Functions Based on Conditioned Spectral Analyses
}

\author{
Wen-Tao Liu ${ }^{1}$ Yun Zhang ${ }^{2} \cdot$ Shou-Ren Wang ${ }^{1}$
}

Received June xx, 202x; revised February xx, 202x; accepted March xx, 202x

(C) Chinese Mechanical Engineering Society and Springer-Verlag Berlin Heidelberg 2017

\begin{abstract}
This paper focuses on the issue of evaluating nonlinear frequency response functions (NOFRFs) of the nonlinear systems with a general input. A new digital approach of evaluating NOFRFs is proposed based on the method of conditioned spectral analysis (CSA). Firstly, a multiple-input/single-output (MISO) linear system with a series of power characterized inputs is obtained by decomposing a nonlinear system according to the NOFRFs theory. Secondly, the correlations among the inputs of various orders are removed by applying CSA method, obtaining an algorithm of identifying the NOFRFs and evaluating the contributions of different order nonlinearities to the output of the system. In the CSA procedure, the different order inputs are conditioned in the sequence from the first order to the highest order. Lastly, two kinds of nonlinear systems with a general input are simulated to verify the effectiveness of the proposed approach on evaluating the frequency response properties of nonlinear systems. It is shown that the results reached by the proposed method are very close to the numerical results obtained by the fourth order Runge-Kutta method, verifying that the proposed method is very effective on the evaluation of NOFRFs.
\end{abstract}

Keywords: Nonlinear system - Frequency response function • Conditioned spectral analysis $\bullet$ System identification

\section{Introduction}

Most practical mechanical systems have nonlinear dynamic characteristics. When a general input is acted on an unknown nonlinear system, the system's nonlinear dynamic behaviors can generate multiplication and intermodulation frequency components in the output

Yun Zhang

zhangyun@mail.tsinghua.edu.cn

1 School of Mechanical Engineering, University of Jinan, Jinan 250022, P.R. China.

2 Beijing Key Lab of Precision/Ultra-precision Manufacturing Equipment and Control, Tsinghua University, Beijing 100084, P.R. China spectra. Due to the fact that the higher order frequencies are much richer than the input frequencies and multiple interferences may occur among all of the frequency components, difficulties are often encountered when one wants to evaluate the nonlinear system's frequency response functions (FRFs) or to quantify the effects of the input on the system.

A common method of analyzing the frequency response properties of nonlinear systems is based on Volterra series [1], which provides a general framework by introducing generalized frequency response functions (GFRFs) [2, 3]. Because the GFRFs represent the nonlinear frequency response features of various orders, the methods of identifying GFRFs has been researched in many studies. One of the identification methods was implemented by minimizing the mean square deviation of output under the condition that the input is a zero-mean stationary Gaussian process [4-5]. But in many mechanical systems, the input does not satisfy Gaussian statistics, such as the vibration excitations generated by raceway geometrical errors in rolling bearings [6]. Therefore, an extended digital method was proposed based on higher order spectra for estimating the linear and quadratic transfer functions of the quadratic time-invariant system with one general input $[7,8]$ and two general inputs [9]. However, for the reason that the number of frequency interactions increases quickly with the order of GFRFs [10-12], explicit expressions of frequency interactions required in the extended approach become very complicated when the order is higher than two or three. Another way to evaluate the GFRFs was by applying parametric modeling techniques, typically involving orthonormal basis expansion [13], analytical derivation [14], and harmonic probing [15-19] which has been applied to detecting cracks in rotor-bearing systems and cantilever beams [20]. Although the methods of para-metric modeling can obtain any order GFRF theoretically, they require not only the output data 
available but also the knowledge of governing motion equations of system, and inherently have the problem of convergence in iteration processes [21, 22].

Recently, a new concept of nonlinear output frequency response functions (NOFRFs) has been proposed by Lang and Billings [23], which allows the analyses of nonlinear systems to be carried out in a manner similar to linear systems and provides a straight forward explanation for the output frequency response properties of nonlinear systems. The effectiveness of NOFRFs has been extensively verified by Peng and Lang etc., involving energy transfer of bilinear oscillators [24], comparison between NOFRFs and harmonic balance of Duffing oscillators [25], parameter estimation of multi-degree-of-freedom (MDOF) nonlinear systems [26] and applications in structural damage detection of nonlinear systems [27] etc. In these studies, however, the NOFRFs are obtained by the algorithm of least square, which requires the system be excited $\mathrm{N}$ times with different input signals and the number of excitations be equal or greater than the highest order of NOFRFs, and thus the algorithm is subject to the constraint that the system input should be controllable either in simulations or in practical experiments.

In this paper, an alternative approach is introduced to evaluate the NOFRFs of the stable time-invariant nonlinear system with a general input. This method is performed by using the technique of conditioned spectral analysis (CSA) which can remove all of the interferences among different order output frequency responses. An algorithm is provided and the contributions of each order nonlinearity to the output are distinguished without requiring that the system be excited by different input signals many times.

\section{The NOFRFs-CSA method}

\subsection{NOFRFs of Nonlinear Systems}

For a stable time-invariant nonlinear system with a general input and a noise contaminated output, the input-output relationship in the neighborhood of an equilibrium point can be expressed by Volterra series as

$$
\left\{\begin{array}{l}
y(t)=\sum_{n=1}^{N} y_{n}(t)+n_{y}(t) \\
y_{n}(t)=\int_{-\infty}^{\infty} \cdots \int_{-\infty}^{\infty} h_{n}\left(\tau_{1}, \cdots, \tau_{n}\right) \prod_{i=1}^{n}\left[x\left(t-\tau_{i}\right) \mathrm{d} \tau_{i}\right]
\end{array}\right.
$$

in which $x(\cdot)$ and $y(\cdot)$ represent the system input and output respectively, $y_{n}(t)$ denotes the output components generated by the $n$th order nonlinearity $(n=1, \cdots, N)$, $h_{n}\left(\tau_{1}, \cdots, \tau_{n}\right)$ is called 'the $n$th order Volterra kernel' or 'the $n$th order impulse response function' which can reduce to the well-known impulse response function of linear systems when $n=1, n_{y}(t)$ denotes the noise imposed on the output, $N$ represents the maximum order of nonlinearities considered for the system. The output components of the orders higher than $N$ are omitted.

In frequency domain, the input-output maps of Eq. (1) can be formulated as [11]

$$
\left\{\begin{array}{l}
Y(\mathrm{j} \omega)=\sum_{n=1}^{N} Y_{n}(\mathrm{j} \omega) \quad \text { for } \forall \omega \\
Y_{n}(\mathrm{j} \omega)=\frac{1}{n^{1 / 2}(2 \pi)^{n-1}} \int_{\omega_{1}+\ldots+\omega_{n}=\omega} Y_{n}\left(\mathrm{j} \omega_{1}, \ldots, \mathrm{j} \omega_{n}\right) \mathrm{d} \sigma_{n \omega}, \\
Y_{n}\left(\mathrm{j} \omega_{1}, \ldots, \mathrm{j} \omega_{n}\right)=H_{n}\left(\mathrm{j} \omega_{1}, \ldots, \mathrm{j} \omega_{n}\right) \prod_{i=1}^{n} X\left(\mathrm{j} \omega_{i}\right)
\end{array}\right.
$$

where $\omega$ signifies the angular frequency, i.e., $\omega=2 \pi f$, $X(\mathrm{j} \omega)$ and $Y(\mathrm{j} \omega)$ represent the system input and output spectra respectively, $Y_{n}(\mathrm{j} \omega)$ denotes the $n$th order output spectral components, $\mathrm{d} \sigma_{n \omega}$ refers to the area of a minute element on the $n$-dimensional hyperplane $\omega=\omega_{1}+\cdots+$ $\omega_{n}, \quad H_{n}\left(\mathrm{j} \omega_{1}, \ldots, \mathrm{j} \omega_{n}\right)$ represents the Generalized Frequency Response functions (GFRFs) equaling to the multidimensional Fourier transform of the $n$th order impulse response $h_{n}\left(\tau_{1}, \cdots, \tau_{n}\right)$, i.e.,

$$
H_{n}\left(\mathrm{j} \omega_{1}, \cdots, \mathrm{j} \omega_{n}\right)=\int_{-\infty}^{\infty} \cdots \int_{-\infty}^{\infty} h_{n}\left(\tau_{1}, \cdots, \tau_{n}\right) \prod_{i=1}^{n}\left[\mathrm{e}^{-\mathrm{j} \omega_{i} \tau_{i}} \mathrm{~d} \tau_{i}\right]
$$

The integration operation in Eq. (2) means that $Y_{n}(\mathrm{j} \omega)$ can be achieved by integrating $Y_{n}\left(\mathrm{j} \omega_{1}, \ldots, \mathrm{j} \omega_{n}\right)$ over a $n$-dimensional hyperplane $\omega=\omega_{1}+\cdots+\omega_{n}$. The GFRFs are nature extensions of the linear frequency response function to the nonlinear systems, because $H_{n}\left(\mathrm{j} \omega_{1}, \ldots, \mathrm{j} \omega_{n}\right)$ is equal to the linear transfer function when $n=1$.

Being different from linear systems, nonlinear systems have more complicated input-output relationship due to the effects of frequency multiplication and intermodulation, giving rise to the difficulties in identifying the contribution of input to output. To address this issue, the concept of NOFRF was proposed by Lang and Billings [23], making the input-output relationship of nonlinear systems much more transparent and physically meaningful.

For a stable time-invariant nonlinear system, the NOFRFs were defined by 


$$
G_{n}(\mathrm{j} \omega)=\frac{\int_{\omega_{1}+\ldots+\omega_{n}=\omega} H_{n}\left(\mathrm{j} \omega_{1}, \ldots, \mathrm{j} \omega_{n}\right) \prod_{i=1}^{n} X\left(\mathrm{j} \omega_{i}\right) \mathrm{d} \sigma_{n \omega}}{\int_{\omega_{1}+\cdots+\omega_{n}=\omega} \prod_{i=1}^{n} X\left(\mathrm{j} \omega_{i}\right) \mathrm{d} \sigma_{n \omega}}
$$

under the condition that

$$
\int_{\omega_{1}+\ldots+\omega_{n}=\omega} \prod_{i=1}^{n} X\left(\mathrm{j} \omega_{i}\right) \mathrm{d} \sigma_{n \omega} \neq 0
$$

where $n=1, \cdots, N$. Based on this, the $n$th order output frequency response of the system are rewritten as

$$
Y_{n}(\mathrm{j} \omega)=G_{n}(\mathrm{j} \omega) X_{n}(\mathrm{j} \omega),
$$

in which

$$
X_{n}(\mathrm{j} \omega)=\frac{1}{n^{1 / 2}(2 \pi)^{n-1}} \int_{\omega_{1}+\cdots+\omega_{n}=\omega} \prod_{i=1}^{n} X\left(\mathrm{j} \omega_{i}\right) \mathrm{d} \sigma_{n \omega} .
$$

Eq. (6) gives a compacted expression for $Y_{n}(\mathrm{j} \omega)$ in a manner similar to the output frequency response of a linear system, where $X_{n}(\mathrm{j} \omega)$ is regarded as the input spectrum

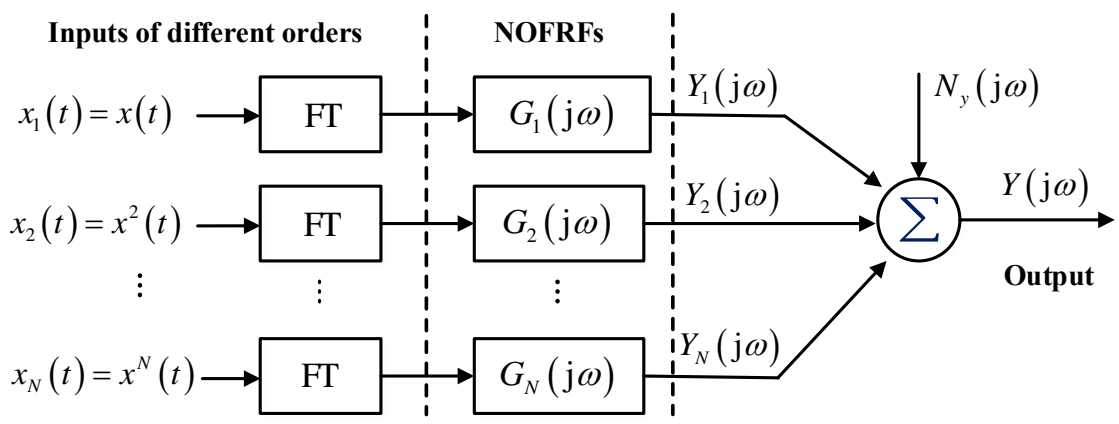

Figure 1 The MISO model of the nonlinear system linearized by NOFRFs

The frequency range of the $n$th order output in Figure 1 is determined by the hyperplane

$$
\omega=\omega_{1}+\omega_{2}+\cdots+\omega_{n},
$$

where $\omega_{i} \in[-b,-a] \cup[a, b], i=1, \cdots, n, \quad 0 \leq a<b$, and $[a, b]$ represents the non-negative frequency range of the original input $x(t)$. In order to identify $\left\{G_{n}(\mathrm{j} \omega)\right\}$ and evaluate the contributions of each order nonlinearity to the output, a digital approach is proposed in the next section based on the CSA technique of MISO linear systems. and $G_{n}(\mathrm{j} \omega)$ is taken as the linear transfer function. Because $X_{n}(\mathrm{j} \omega)$ can be formulated as the Fourier transform of the time sequence $x^{n}(t)$, that is,

$$
X_{n}(\mathrm{j} \omega)=\frac{1}{\sqrt{2 \pi}} \int_{-\infty}^{\infty} x^{n}(t) \mathrm{e}^{-\mathrm{j} \omega t} \mathrm{~d} t
$$

$x^{n}(t)$ can be seen as the input signals of the linear system $G_{n}(\mathrm{j} \omega)$ in time domain.

Substituting Eq. (6) into Eq. (2) gives

$$
Y(\mathrm{j} \omega)=\sum_{n=1}^{N} G_{n}(\mathrm{j} \omega) X_{n}(\mathrm{j} \omega),
$$

which reflects that the nonlinear system under study can be decomposed into a set of linear systems. Therefore, the input-output relationship of the nonlinear system can be illustrated by a multiple-input/single-output (MISO) linear system as plotted in Figure 1. $\left\{x^{n}(t)\right\}$ are the inputs and $\left\{G_{n}(\mathrm{j} \omega)\right\}$ represent the linear transfer functions of the equivalent linear system. It should be noted that $\left\{G_{n}(\mathrm{j} \omega)\right\}$ depend not only on the nonlinearity of the system but also on the inputs as expressed in Eq. (4).

\subsection{Application of CSA in Evaluating NOFRFs}

As for the MISO linear system, the input-output relationship can be quantified by the ordinary coherence function

$$
\gamma_{i y}^{2}(\mathrm{j} \omega)=\frac{\left|S_{i y}(\mathrm{j} \omega)\right|^{2}}{S_{i i}(\mathrm{j} \omega) S_{y y}(\mathrm{j} \omega)}
$$

under the condition that the inputs are uncorrelated, where $S_{i i}(\mathrm{j} \omega), S_{y y}(\mathrm{j} \omega)$ and $S_{i y}(\mathrm{j} \omega)$ represent the auto-power and cross-power spectra and of the $i$ th input and the output respectively. In the study, however, the inputs of the equivalent MISO linear system may be correlated with 
each other, resulting in the difficulties in distinguishing the effects of different order nonlinearities to the output.

Conditioned spectral analysis provide a feasible way for solving the correlation problem of MISO linear systems, but it cannot be applied directly to nonlinear systems because the multiplication and intermodulation components of frequency are not taken into account in the existing CSA theories. Based on the NOFRFs model, the CSA technique will be extended to nonlinear systems in the study.

As shown in Figure 1, a stable time-invariant nonlinear system with a general input can be decomposed into a MISO linear system near an equilibrium point. According to the procedure of CSA, the correlations between the inputs of different orders can be removed by taking conditioning operations, obtaining an equivalent MISO linear system with multiple uncorrelated inputs as shown in Figure 2. $\left\{X_{i \cdot(i-1) !}(\mathrm{j} \omega), i=1, \cdots, N+1\right\}$ represent the conditioned inputs, that is, the $i$ th order input is conditioned by all of the lower order inputs $\left\{X_{1}(\mathrm{j} \omega), X_{2}(\mathrm{j} \omega), \cdots, X_{i-1}(\mathrm{j} \omega)\right\}$ in sequence, meaning that the parts of $X_{i}(\mathrm{j} \omega)$ correlated with the lower order inputs are all removed. Thus, the conditioned inputs are uncorrelated with each other. The first order input $X_{1}(\mathrm{j} \omega)$ can be regarded as $X_{1 \cdot 0 !}(\mathrm{j} \omega)$ where 0 ! denotes no conditions are operated. The last conditioned input $X_{(N+1) \cdot N !}(\mathrm{j} \omega)$ refers to the measured output conditioned by the inputs $\left\{X_{i}(\mathrm{j} \omega), i=1,2, \cdots, N\right\}$, equaling to the spectrum of the noise $n_{y}(t)$ in Figure 1.

It is worth noting that the inputs are conditioned here from the first order to highest order, that is, the first input in Figure 2 is identical with the first one in Figure 1, and the $i$ th $(i>1)$ conditioned input equals to the $i$ th order input in Figure 1 conditioned by the lower orders. Obviously, conditioning in this sequence conforms to the physical meaning of Volterra series model of the nonlinear system, because the $i$ th input in Figure 1 depends on the order of frequency intermodulation and the higher order intermodulation is based on the intermodulation of lower orders.

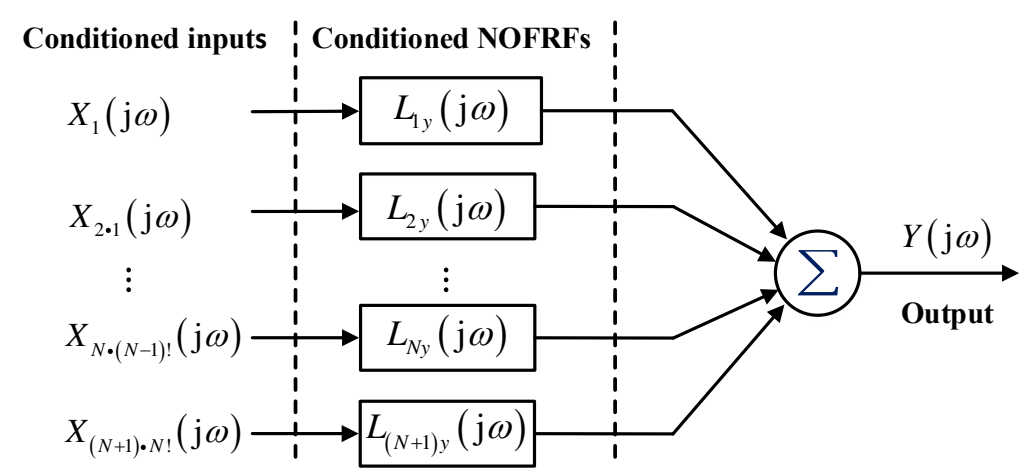

Figure 2 The CSA model of the nonlinear system linearized by NOFRFs.

$\left\{L_{i y}(\mathrm{j} \omega), i=1,2, \cdots, N+1\right\}$ in Figure 2 represent the linear transfer functions between the $i$ th order conditioned input and the output. $L_{(N+1) y}(\mathrm{j} \omega)$ equals to 1 according to the meaning of $X_{(N+1) \cdot N !}(\mathrm{j} \omega)$. Noted that although $\left\{L_{i y}(\mathrm{j} \omega)\right\}$ are quite different from the NOFRFs $\left\{G_{i}(\mathrm{j} \omega)\right\}$ except $L_{1 y}(\mathrm{j} \omega)=G_{1}(\mathrm{j} \omega)$, the contributions of the inputs to the output are not essentially influenced by the transforming. The relationship between the two models can be formulated as [28]

$$
L_{i y}(\mathrm{j} \omega)=\sum_{j=i}^{N} L_{i j}(\mathrm{j} \omega) G_{j}(\mathrm{j} \omega)
$$

in which $i=1,2, \cdots, N$, and $L_{i j}(\mathrm{j} \omega)$ represents the linear transfer function between the conditioned inputs
$X_{i \cdot(i-1) !}(\mathrm{j} \omega)$ and $X_{j \cdot(i-1) !}(\mathrm{j} \omega)$. It is supposed that $L_{i j}(\mathrm{j} \omega)=0$ when $j>i$ and $L_{i j}(\mathrm{j} \omega)=1$ when $j=i$.

The conditioned inputs $\left\{X_{i \cdot(i-1) !}(\mathrm{j} \omega), 2 \leq i \leq N+1\right\}$ are formulated as

$$
X_{i \bullet(i-1) !}(\mathrm{j} \omega)=X_{i}(\mathrm{j} \omega)-\sum_{k=1}^{i-1} L_{k i}(\mathrm{j} \omega) X_{k \bullet(k-1) !}(\mathrm{j} \omega)
$$

which can be implemented by the iteration

$$
X_{i \bullet k !}(\mathrm{j} \omega)=X_{i \bullet(k-1) !}(\mathrm{j} \omega)-L_{k i}(\mathrm{j} \omega) X_{k \bullet(k-1) !}(\mathrm{j} \omega)
$$

where $1 \leq k \leq i-1$. The conditioned linear transfer functions $\left\{L_{i j}(\mathrm{j} \omega)\right\}$ are given by 


$$
L_{i j}(\mathrm{j} \omega)=\frac{S_{i j \cdot(i-1) !}(\mathrm{j} \omega)}{S_{i i \cdot(i-1) !}(\mathrm{j} \omega)},
$$

in which $1 \leq i<j \leq N+1, \quad L_{i(N+1)}(\mathrm{j} \omega)$ refers to $L_{i y}(\mathrm{j} \omega), S_{i i \cdot(i-1) !}(\mathrm{j} \omega)$ represents the auto-power spectral density of the conditioned input $X_{i \cdot(i-1) !}(\mathrm{j} \omega)$, and $S_{i j \cdot(i-1) !}(\mathrm{j} \omega)$ the cross-power spectral density of the conditioned inputs $X_{i \cdot(i-1) !}(\mathrm{j} \omega)$ and $X_{j \cdot(i-1) !}(\mathrm{j} \omega)$. The power spectral densities are defined by

$$
S_{i j \cdot(i-1) !}(\mathrm{j} \omega)=\mathrm{E}\left[X_{i \cdot(i-1) !}^{*}(\mathrm{j} \omega) X_{j \cdot(i-1) !}(\mathrm{j} \omega)\right]
$$

and

$$
S_{i i \bullet(i-1) !}(\mathrm{j} \omega)=\mathrm{E}\left[X_{i \bullet(i-1) !}^{*}(\mathrm{j} \omega) X_{i \bullet(i-1) !}(\mathrm{j} \omega)\right]
$$

respectively, where $\mathrm{E}[\cdot]$ denotes the value of expectation and the superscript ${ }^{*}$ signifies complex conjugation. The conditioned input power spectra can be obtained by

$$
S_{i j \bullet k !}(\mathrm{j} \omega)=S_{i j \cdot(k-1) !}(\mathrm{j} \omega)-L_{k j}(\mathrm{j} \omega) S_{i k \cdot(k-1) !}(\mathrm{j} \omega),
$$

and in the case of $i=j$,

$$
S_{i i \bullet k !}(\mathrm{j} \omega)=S_{i i \cdot(k-1) !}(\mathrm{j} \omega)-\left|L_{k i}(\mathrm{j} \omega)\right|^{2} S_{k k \cdot(k-1) !}(\mathrm{j} \omega) \text {. }
$$

Iterating Eqs. (15), (18) and (19) from the first order to the maximal order yields the optimum estimations of $\left\{L_{i j}(\mathrm{j} \omega)\right\},\left\{S_{i j \cdot k !}(j \omega)\right\}$ and $\left\{S_{i i \cdot k !}(j \omega)\right\}$.

Given the conditioned input-output transfer functions $\left\{L_{i y}(\mathrm{j} \omega)\right\}$, the NOFRFs $\left\{G_{i}(\mathrm{j} \omega)\right\}$ can be deduced from Eq. (12), i.e.,

$$
\left\{\begin{array}{l}
G_{N}(\mathrm{j} \omega)=L_{N y}(\mathrm{j} \omega) \\
G_{i}(\mathrm{j} \omega)=L_{i y}(\mathrm{j} \omega)-\sum_{j=i+1}^{N} L_{i j}(\mathrm{j} \omega) G_{j}(\mathrm{j} \omega)
\end{array}\right.
$$

in which $i=N-1, N-2, \cdots, 1$. Eq. (20) provides an alternative way for evaluating the NOFRFs of the nonlinear system.

To sum up, the proposed digital method of evaluating NOFRFs of the nonlinear systems with a general input is carried out by the algorithm as shown in Figure 3 .

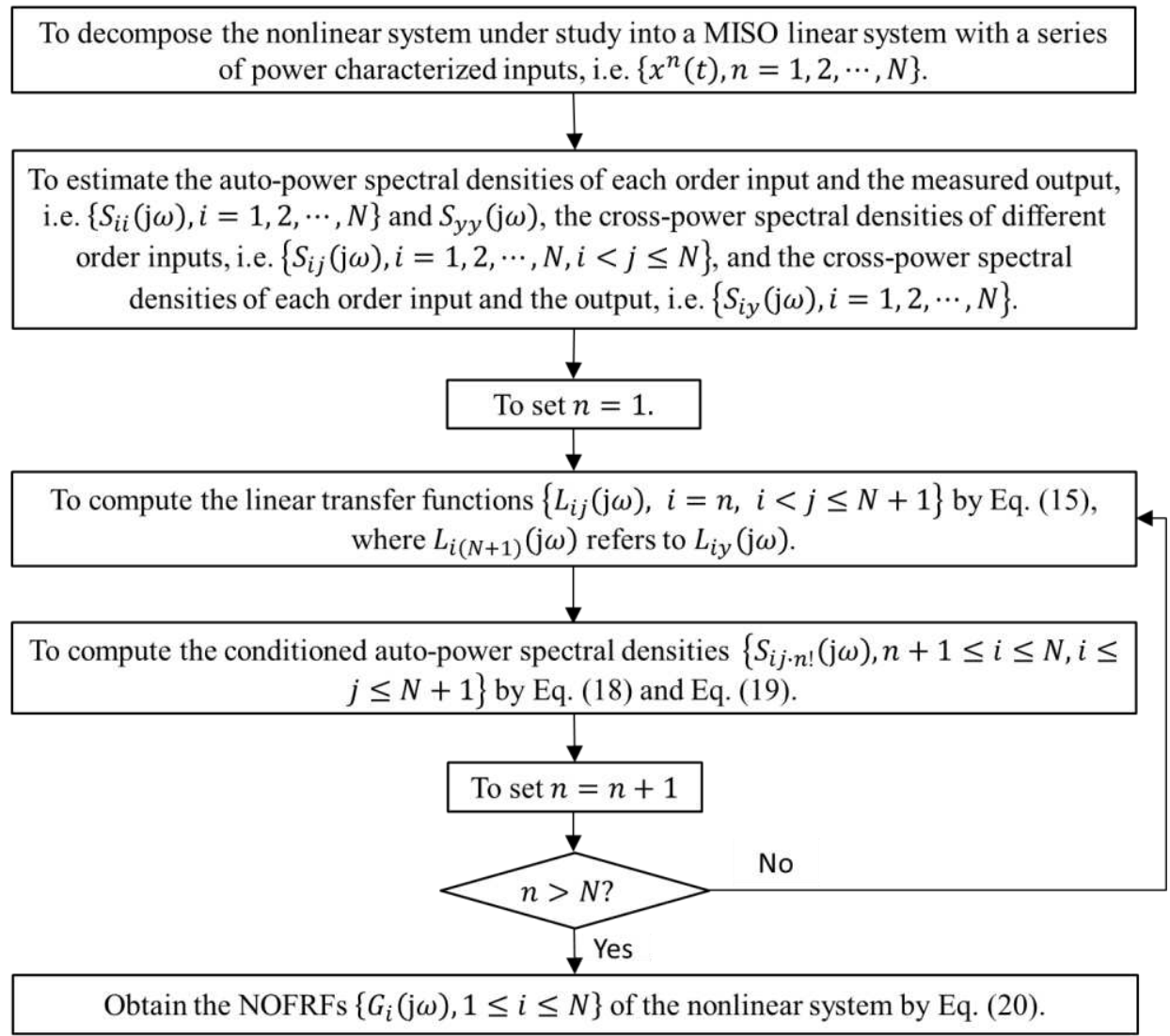

Figure 3 The proposed algorithm of evaluating NOFRFs based on CSA. 
As a result, the output spectra generated by each order unconditioned input can be yielded by Eq. (6), such that the contributions of each order nonlinearity to the output are distinguished by this way. In addition, the total output power spectrum of the nonlinear system responding to the original input $x(t)$ is given by

$$
S_{y: x}(\mathrm{j} \omega)=\sum_{i=1}^{N}\left|L_{i y}(\mathrm{j} \omega)\right|^{2} S_{i i \bullet(i-1) !}(\mathrm{j} \omega)
$$

and the contribution ratio of the original input to the measured output can be quantified by

$$
\gamma_{x y}^{2}(\mathrm{j} \omega)=\sum_{i=1}^{N} \frac{S_{y: i \cdot(i-1) !}(\mathrm{j} \omega)}{S_{y y}(\mathrm{j} \omega)}
$$

\section{Simulation studies}

In order to verify the effectiveness of the proposed method, two kinds of nonlinear systems are simulated in this section.

\subsection{The first nonlinear system}

The first nonlinear system is a known mechanical oscillator as shown in Figure 4. Its motion equation is

$$
\left\{\begin{array}{l}
m \frac{d^{2} y_{0}(t)}{d t^{2}}+c \frac{d y_{0}(t)}{d t}+k_{1} y_{0}(t)+k_{2} y_{0}^{2}(t)+k_{3} y_{0}^{3}(t)=x(t) \\
y(t)=y_{0}(t)+n(t)
\end{array},\right.
$$

where $x(t)$ is the force acted on the mass $m, y_{0}(t)$ is the displacement of the mass, $y(t)$ represents the measured displacement of the mass, $n(t)$ denotes a zero-mean Gaussian noise imposed on the measured data of $y_{0}(t), m=1 \mathrm{~kg}, c=20 \mathrm{~N} \cdot \mathrm{s} \cdot \mathrm{m}^{-1}, k_{1}=10^{4} \mathrm{~N} \cdot \mathrm{m}^{-1}$, $k_{2}=10^{7} \mathrm{~N} \cdot \mathrm{m}^{-2}$ and $k_{3}=5 \times 10^{9} \mathrm{~N} \cdot \mathrm{m}^{-3}$ are the characteristic parameters of the system [28].

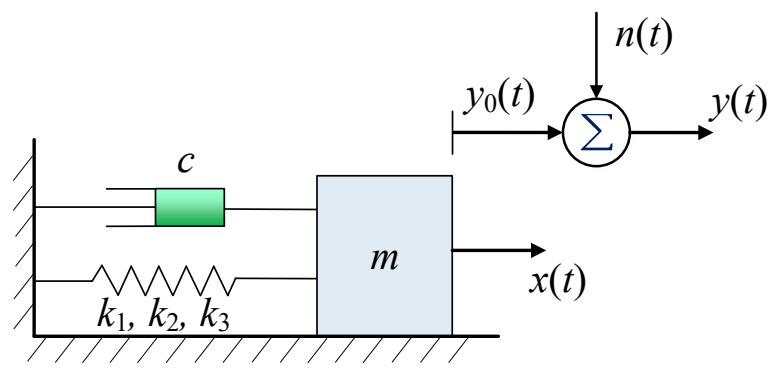

Figure 4 The first nonlinear system for simulation
The general input of the system is given by

$$
x(t)=\frac{3}{2 \pi} \frac{\sin (2 \times 55 \times \pi \times t)-\sin (2 \times 30 \times \pi \times t)}{t},
$$

in which $-2.56 \leq t \leq 2.56$ seconds as plotted in Figure 5(a). The output of the system is numerically simulated by using the fourth order Runge-Kutta method, as shown in Figure 5(b), and the input and output amplitude spectra obtained by FFT are sketched in Figure 6 . The output spectrum distributes in the lower range of $0 \sim 20 \mathrm{~Hz}$ besides the input frequency range of $30 \sim 55 \mathrm{~Hz}$, particularly near $15 \mathrm{~Hz}$ where a distinct peak (i.e., the peak A) appears, implying that a part of power is transferred to the lower frequency range.
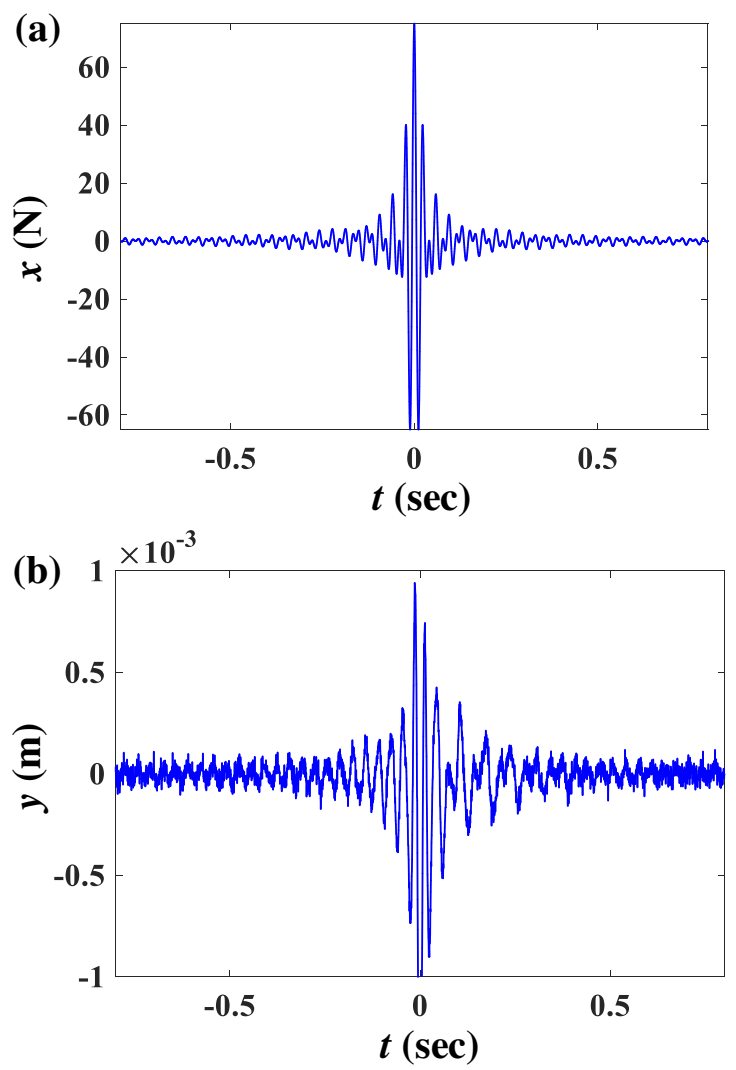

Figure 5 The time sequences of the system input and output: (a) the input, (b) the output. 

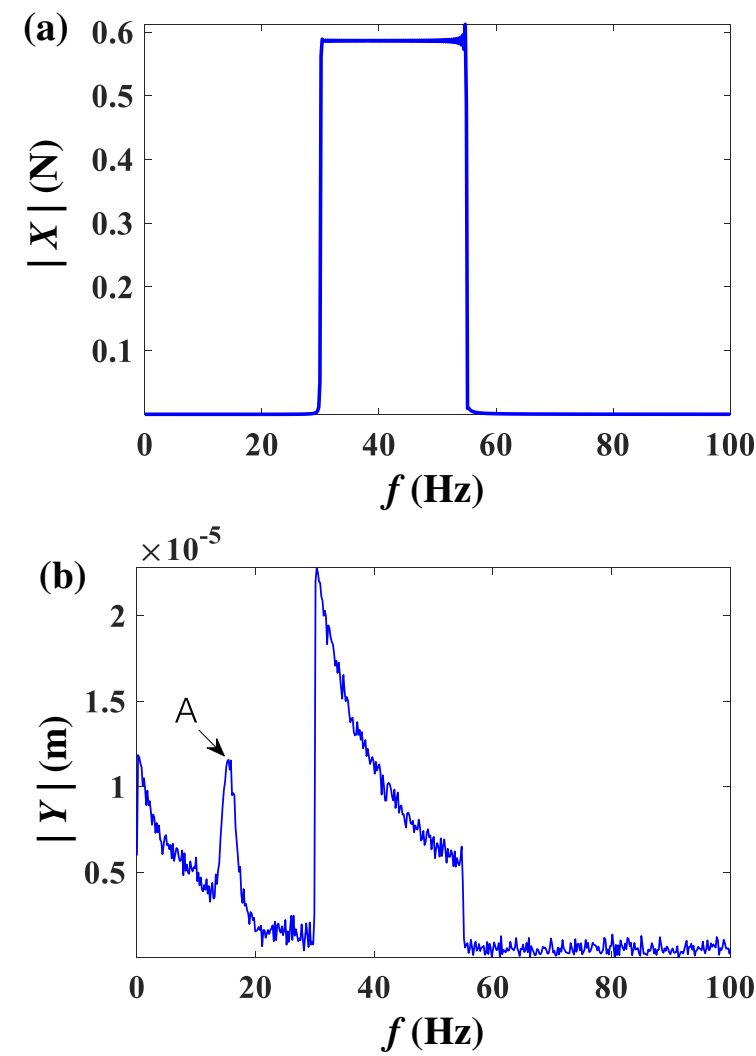

Figure 6 The amplitude spectra of the system input and output obtained by FFT: (a) the input, (b) the output.

\subsubsection{Correlations of the input between different orders}

Given the time sequence of the input, the higher order input spectra $\left\{X_{n}(\mathrm{j} \omega)\right\}$ in Eq. (9) can be yielded by taking Fourier transform of the power series $x^{n}(t)$. For example, the amplitude spectra of the first five order inputs obtained by FFT, i.e. $\left\{\left|X_{n}(\mathrm{j} \omega), n=1, \cdots, 5\right|\right\}$, are as plotted in Figure 7. The magnitude and distribution of the five spectra are different obviously. Both the number and magnitude of crests increase with the order of input, and the input frequency range also widens with orders.

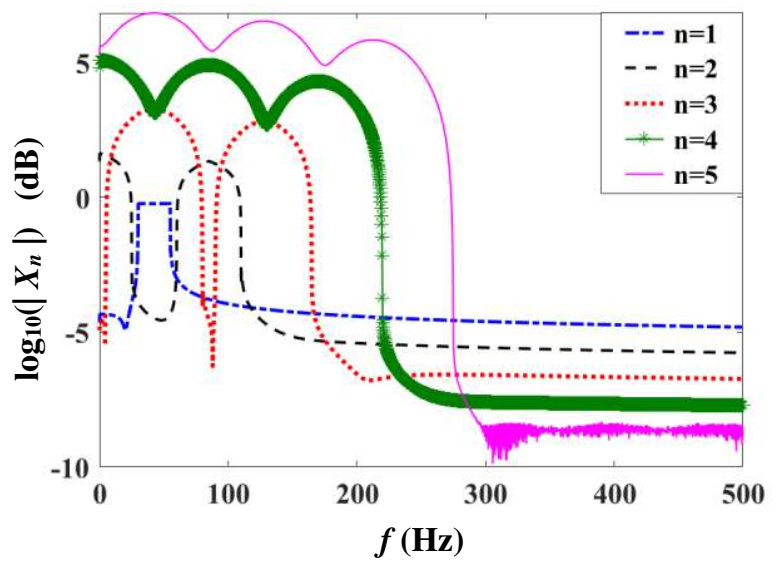

Figure 7 The amplitude spectra of the first five order inputs obtained by FFT

In order to determine whether there are correlations between the inputs of different orders, the ordinary coherence function $\gamma_{i j}^{2}(\omega)=\left|S_{i j}(\mathrm{j} \omega)\right|^{2}\left[S_{i i}(\mathrm{j} \omega) S_{j j}(\mathrm{j} \omega)\right]^{-1}$ is applied, where $S_{i j}(\mathrm{j} \omega)$ represents the cross-spectral density functions of the $i$ th and $j$ th order inputs, $S_{i i}(\mathrm{j} \omega)$ and $S_{j j}(\mathrm{j} \omega)$ represent the auto-power spectral density functions respectively. The results are as plotted in Figure 8 , which indicate that there exist significant correlations between the inputs of different orders. Take $\left\{\gamma_{1 j}^{2}, j=\right.$ $2,3,4,5\}$ for example, as shown in Figure 8(a), $x_{1}(t)$ and $x_{2}(t)$ are highly correlated in the two narrow bands, i.e. $24 \sim 30 \mathrm{~Hz}$ and $55 \sim 62 \mathrm{~Hz}$, while $x_{1}(t)$ and $x_{3}(t)$ have correlation in the lower frequency range of $0 \sim 13 \mathrm{~Hz}$ and the higher frequency range of $70 \sim 100 \mathrm{~Hz}$, and $x_{1}(t)$ and $x_{4}(t)$ have notable correlation in the middle range of $30 \sim 55 \mathrm{~Hz}$ where the correlation between $x_{1}(t)$ and $x_{5}(t)$ is even higher. Besides, the correlation between $x_{1}(t)$ and $x_{5}(t)$ is remarkable in the ranges of $0 \sim 10 \mathrm{~Hz}$ and $77 \sim 96 \mathrm{~Hz}$. Similarly, distinct correlations also exist between the 2nd, 3rd and 4th order inputs, which can be seen in Figure $8(\mathrm{~b}) \sim(d)$, respectively.

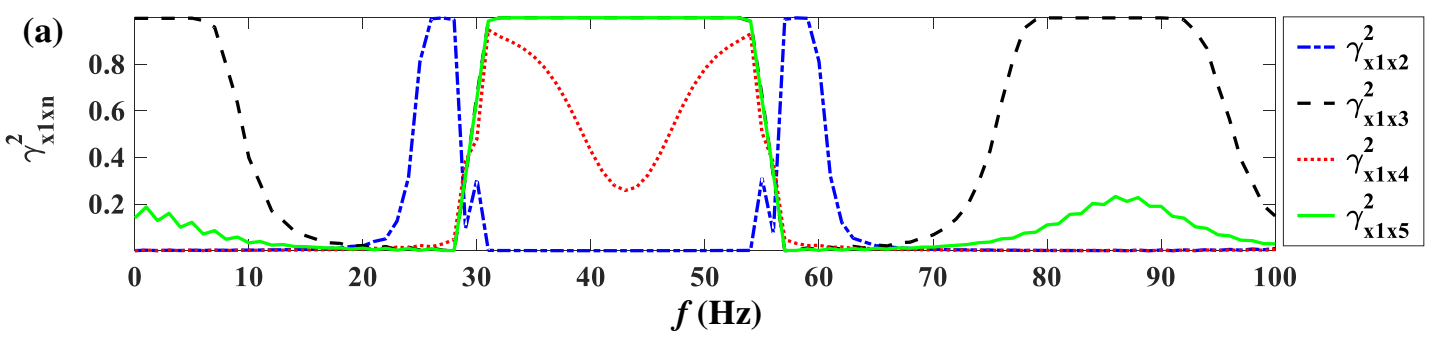



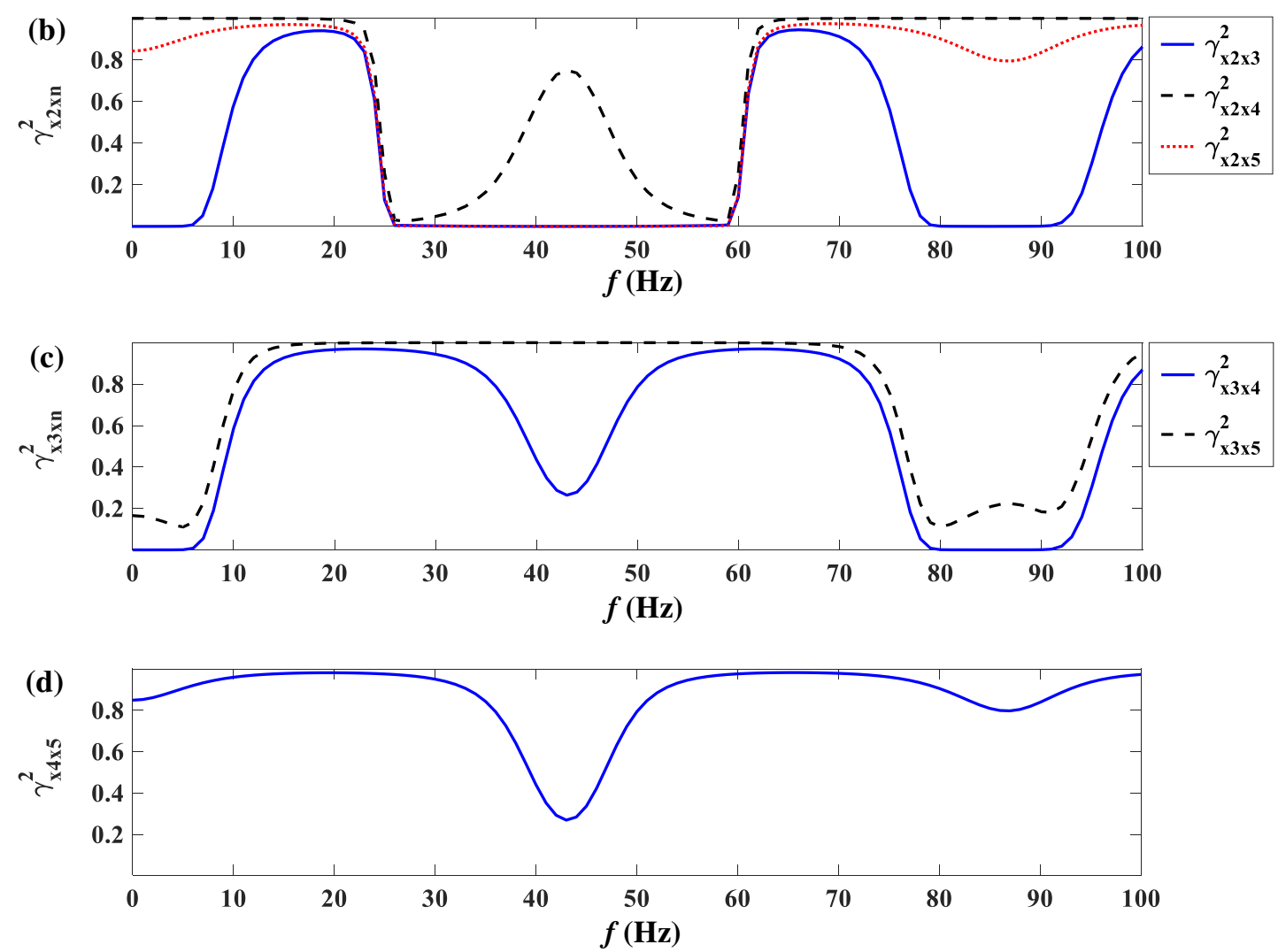

Figure 8 The coherence spectra of the input between different orders: (a) between the first and the higher orders, (b) between the second and the higher orders, (c) between the third and the higher orders, (d) between the fourth and the fifth orders.

\subsubsection{Nonlinear output response analysis based on CSA method}

To reveal the contributions of each order nonlinearity to the system output, CSA technique is applied here. The conditioned input amplitude spectra $\left\{\left|X_{i \cdot(i-1) !}(\mathrm{j} \omega)\right|, i=\right.$ $1,2, \cdots, 5\}$ are shown in Figure 9, between which the correlations are all removed. The output power spectral components corresponding to the conditioned inputs are plotted in Figure 10(a) (e), and the summation $S_{y: x}=$ $\sum_{i=1}^{5} S_{y: i \cdot(i-1) !}$ is displayed in Figure 10(f). It is illustrated that the output component correlated with the first order input is prominent in the frequency range of $30 \sim 55 \mathrm{~Hz}$, while the output component correlated with the second order conditioned input is dominated in the lower frequency range of $0 \sim 20 \mathrm{~Hz}$. Nevertheless, it cannot be stated by now that the output power distributed in $30 \sim$ $55 \mathrm{~Hz}$ is generated mainly by the first order input, because all of the correlated output components induced actually by the second to fifth order nonlinearities of the system are attributed to the first order in the CSA procedure. Similarly, it cannot be concluded until now that the transferred power in $\quad 0 \sim 20 \mathrm{~Hz}$ is generated mainly by the second order conditioned input, because all of the correlated output components induced actually by the third to fifth order nonlinearity are also attributed to the second order in the lower frequency range in the method.

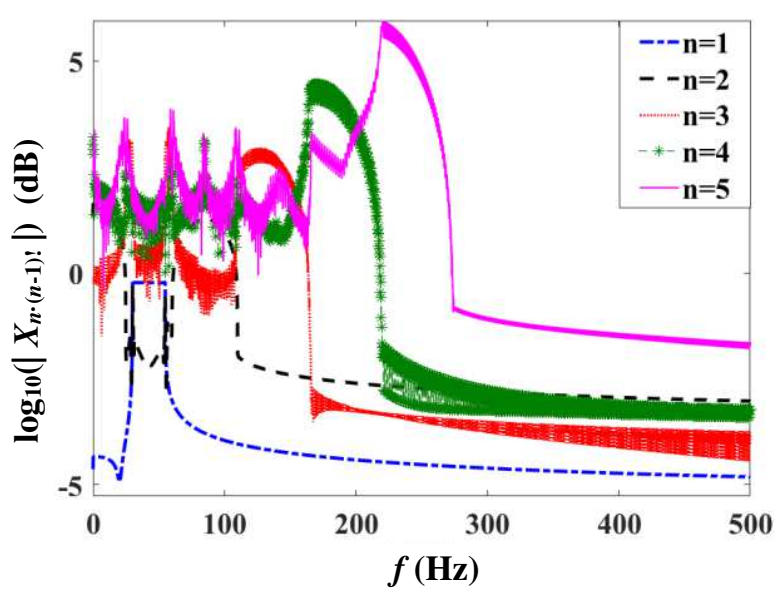

Figure 9 The amplitude spectra of the conditioned inputs 

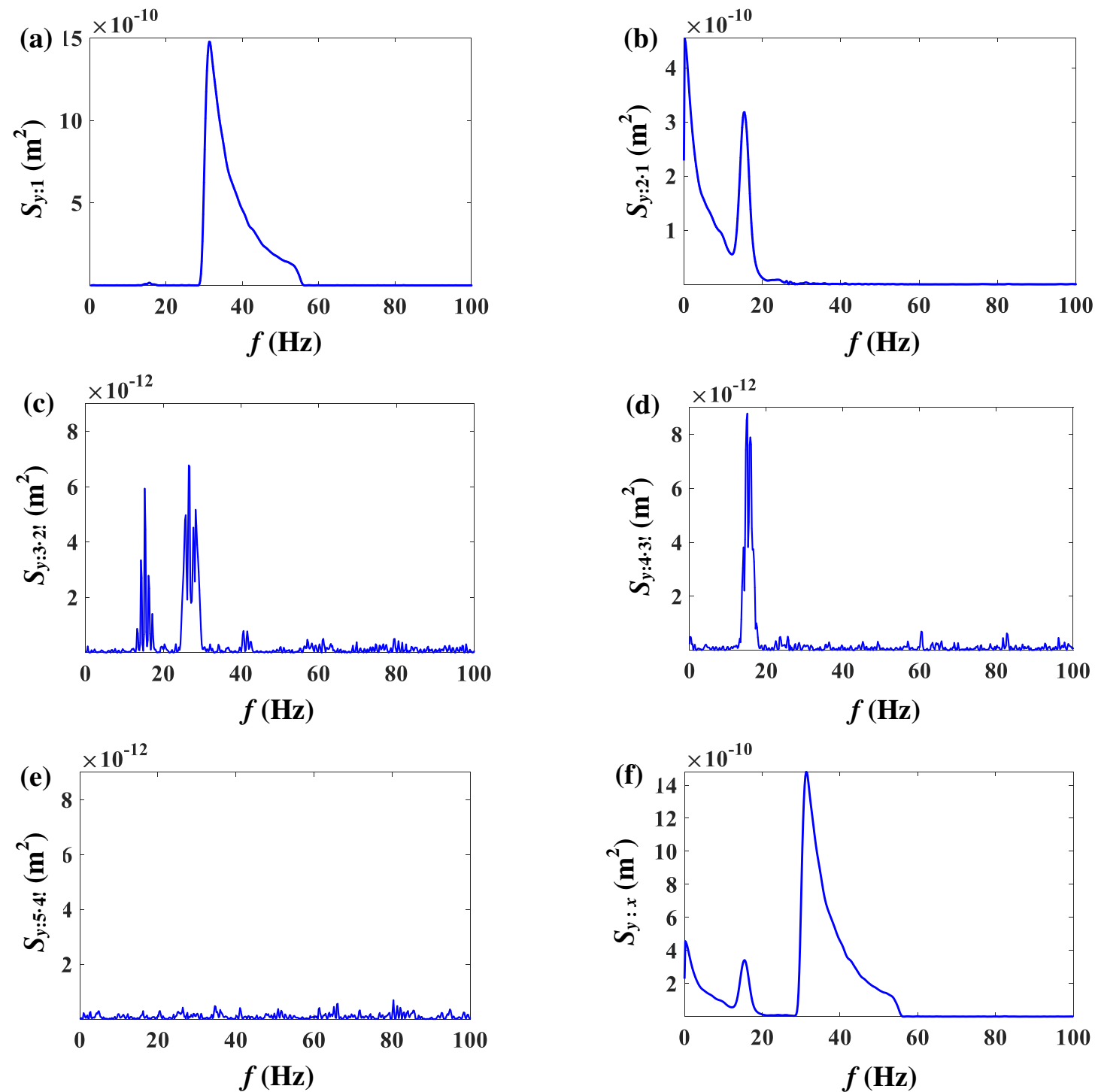

Figure 10. The power spectra of the first five conditioned outputs and their summation of the first nonlinear system: (a)

$$
S_{y: 1}(f) \text {, (b) } S_{y: 2 \cdot 1}(f) \text {, (c) } S_{y: 3 \cdot 2 !}(f) \text {, (d) } S_{y: 4 \cdot 3 !}(f) \text {, (e) } S_{y: 5 \cdot 4 !}(f) \text {, (f) } S_{y: x}(f) \text {. }
$$

The first five order NOFRFs $\left\{G_{i}(\mathrm{j} \omega), i=1,2, \cdots, 5\right\}$ of the nonlinear system are obtained by Eq. (24), and their amplitude spectra $\left\{\left|G_{i}(\mathrm{j} \omega)\right|\right\}$ are plotted in Figure 11, which reflect the amplitude-frequency response properties of each order nonlinearity respecting the specific input. The second to fifth order NOFRFs all have higher amplitudes in the frequency range of $30 \sim 55 \mathrm{~Hz}$, meaning that the nonlinear system has great gains on the amplitudes of the second to fifth order unconditioned inputs in the frequency range. Besides, both $\left|G_{3}(\mathrm{j} \omega)\right|$ and $\left|G_{5}(\mathrm{j} \omega)\right|$ exhibit many distinct peaks in the lower frequency range of $0 \sim 20 \mathrm{~Hz}$, implying that the system also have notable gains on the amplitudes of the third and fifth order unconditioned inputs in the lower range. Nevertheless, because the $i$ th order output frequency response is equal to the product of $G_{i}(\mathrm{j} \omega)$ and $X_{i}(\mathrm{j} \omega)$ as expressed by Eq. (6), the nonlinear output frequency responses of each order depends not merely on the NOFRF but also on the input. 

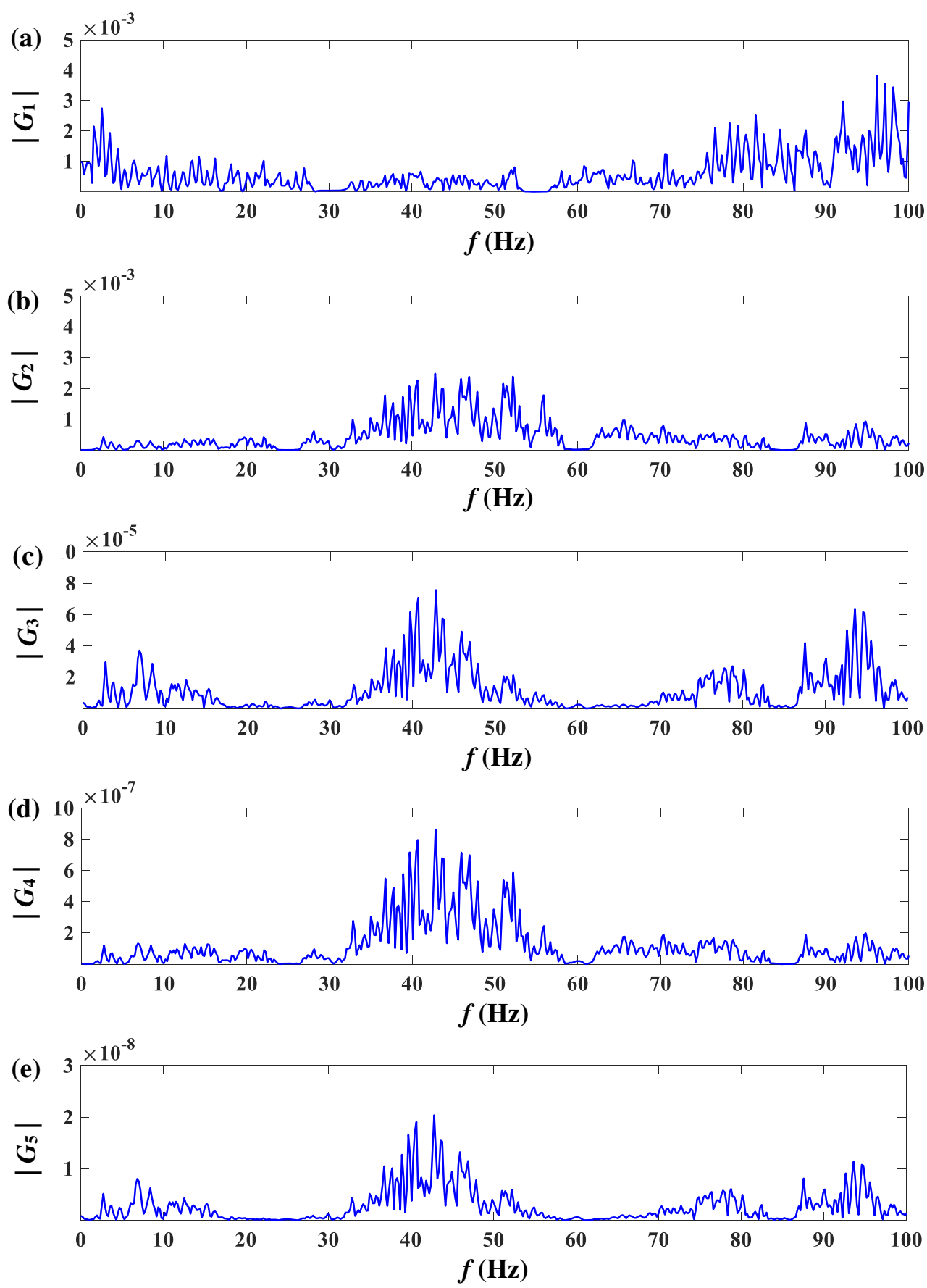

Figure 11 The amplitude spectra of the first five order NOFRFs of the first nonlinear system: (a) $\left|G_{1}(f)\right|$; (b) $\left|G_{2}(f)\right|$; (c) $\left|G_{3}(f)\right|$; (d) $\left|G_{4}(f)\right| ;\left(\right.$ e) $\left|G_{5}(f)\right|$.

By Eq. (6) one can obtain exactly the output spectra of the nonlinear system responding to each order unconditioned input, which reveal the contributions of each order nonlinearity to the system output without considering interferences. The amplitude spectra of the unconditioned output responses, i.e. $\left\{\left|Y_{i}(\mathrm{j} \omega)\right|, i=1, \cdots, 5\right\}$, are plotted in Figure 12(a) (e). The first, third and fifth order inputs induce remarkable output responses in the frequency range of $30 \sim 55 \mathrm{~Hz}$ which is identical with the first order input frequency range, while the second and fourth order inputs mainly give rise to the output components in the lower frequency range and the higher frequency range. 

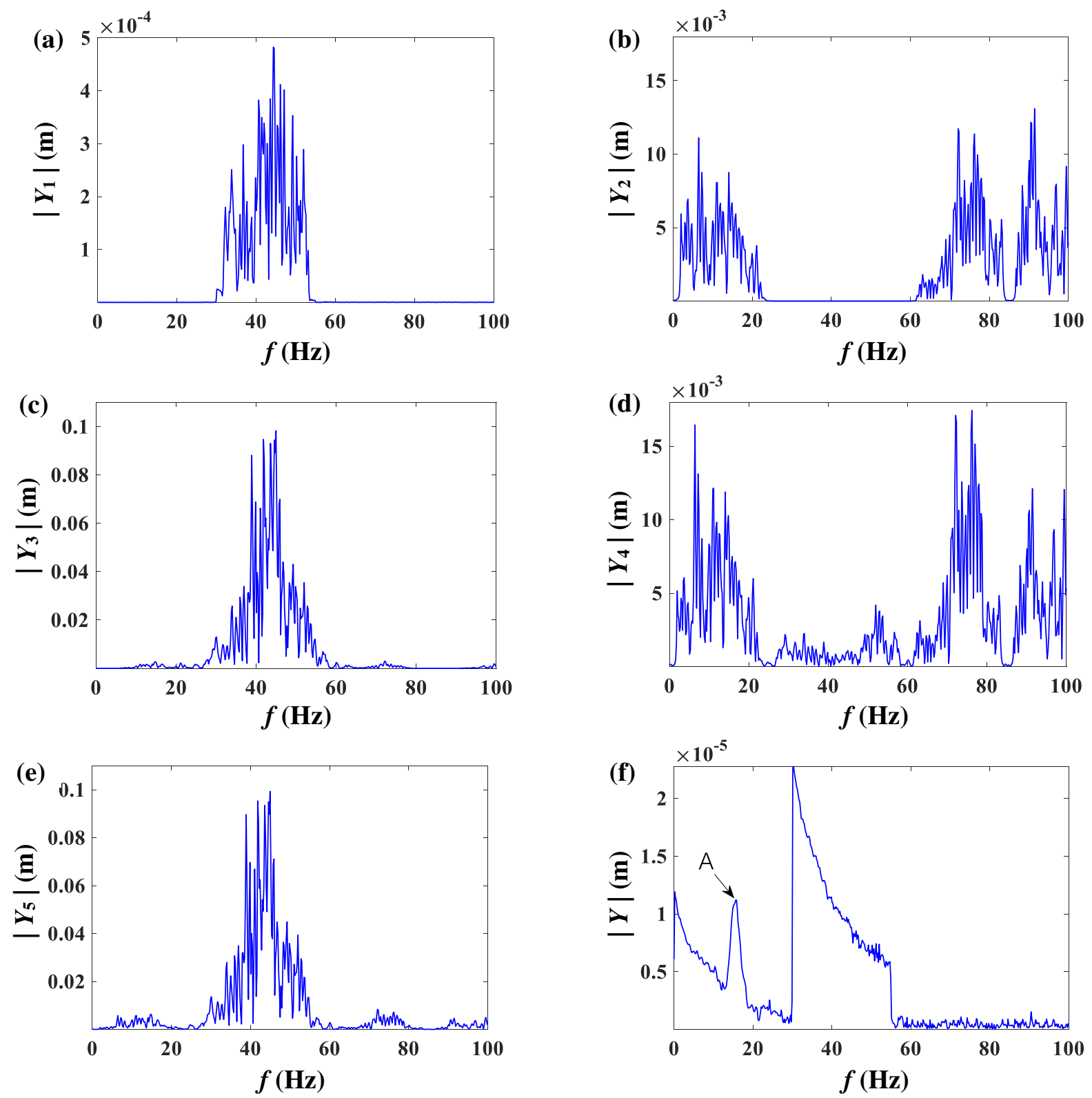

Figure 12 The amplitude spectra of the first five order unconditioned output components of the first nonlinear system and the summing spectrum of them: (a) $\left|Y_{1}(f)\right|$; (b) $\left|Y_{2}(f)\right|$; (c) $\left|Y_{3}(f)\right|$; (d) $\left|Y_{4}(f)\right|$; (e) $\left|Y_{5}(f)\right|$; (f) $|Y(f)|$.

The amplitude spectrum of total output yielded by summing the unconditioned output response components, i.e. $\left|\sum_{i=1}^{5} Y_{i}(\mathrm{j} \omega)\right|$, is shown in Figure 12(f), which matches the result of Runge-Kutta method in Figure 6(b) very well, verifying numerically that the CSA based method is effective in distinguishing the contributions of each order nonlinearity to the output of the system. For example, at the frequency of $15.64 \mathrm{~Hz}$ where the distinct peak A takes place in the range of $10 \sim 20 \mathrm{~Hz}$, the first five order responses and their summation are listed in Table 1. The magnitude of the first order output response at the frequency is only $5.4055 \times 10^{-9} \mathrm{~m}$ which is much smaller than other orders, so its influence on the output is slight. Although the magnitudes of the second and fourth order responses are higher at the frequency, but their phases are inverse, leading to the reduction amplitude. For the same reason, the output responses of the third and fifth orders are also cut down. As a result, the magnitude of the summation of the first five order output responses is equal to $1.1299 \times 10^{-5} \mathrm{~m}$ which is close to the numerical result $1.0993 \times 10^{-5} \mathrm{~m}$ obtained by Runge-Kutta method. The deviation of $2.784 \%$ is mainly due to the noise $n_{y}(t)$ imposed on the output, which is common in practical 
systems.

Table 1 The contributions of the first five order nonlinearities to the output of the first nonlinear system and their summation at the frequency of $15.64 \mathrm{~Hz}$

\begin{tabular}{ll}
\hline \multicolumn{1}{c}{ Items } & \multicolumn{1}{c}{ Values } \\
\hline$Y_{1}(f)$ & $1.6873 \times 10^{-9}-5.1354 \times 10^{-9} \mathrm{j}$ \\
$Y_{2}(f)$ & $0.0025-0.0023 \mathrm{j}$ \\
$Y_{3}(f)$ & $5.1129 \times 10^{-4}+6.4689 \times 10^{-4} \mathrm{j}$ \\
$Y_{4}(f)$ & $-0.0027+0.0030 \mathrm{j}$ \\
$Y_{5}(f)$ & $-0.0004-0.0014 \mathrm{j}$ \\
$\sum_{n=1}^{5} Y_{n}(f)$ & $-1.1106 \times 10^{-5}-2.0774 \times 10^{-6} \mathrm{j}$ \\
$\left|\sum_{n=1}^{5} Y_{n}(f)\right|$ & $1.1299 \times 10^{-5}$ \\
\hline
\end{tabular}

\subsection{The second nonlinear system}

The second case for our simulation is a quadratic system with time lags as shown in Figure 13. Its governing equation is given by

$$
\left\{\begin{array}{l}
y_{0}(t)=k_{1} x(t)+k_{2} x\left(t-\tau_{1}\right)+k_{3} x^{2}(t)+k_{4} x^{2}\left(t-\tau_{2}\right) \\
y(t)=y_{0}(t)+n(t)
\end{array},\right.
$$

where $x(t)$ and $y_{0}(t)$ represent the input and output of the system in time domain respectively, $y(t)$ is the measured output of the system, $n(t)$ denotes a zero-mean Gaussian noise acted on the output, $k_{1}=-0.64, k_{2}=$ $1.0, \quad k_{3}=0.9$ and $k_{4}=1.0$ are characteristic parameters, $\tau_{1}=0.001 \mathrm{~s}$ and $\tau_{2}=0.0005 \mathrm{~s}$ refer to the time delays. Suppose that both input and output of the system are displacements and the input is formed by Eq. (24) as same as the first nonlinear system.

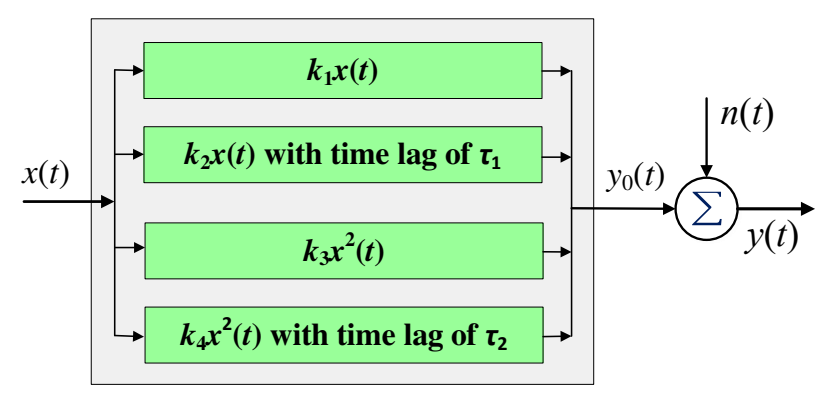

Figure 13 The quadratic nonlinear system with time lags for simulation

The output sequence of time obtained directly by Eq. (25) is shown in Figure 14(a), and the amplitude spectrum of the output obtained by FFT is sketched in Figure 14(b). Obviously, the magnitude of the output spectrum is prominent in the two ranges of $0 \sim 25 \mathrm{~Hz}$ and $60 \sim 110 \mathrm{~Hz}$, while in the frequency range of input, i.e., $30 \sim 55 \mathrm{~Hz}$, the magnitude is approximately equal to zero, implying that almost all of the power is transferred to the lower and higher frequency ranges.
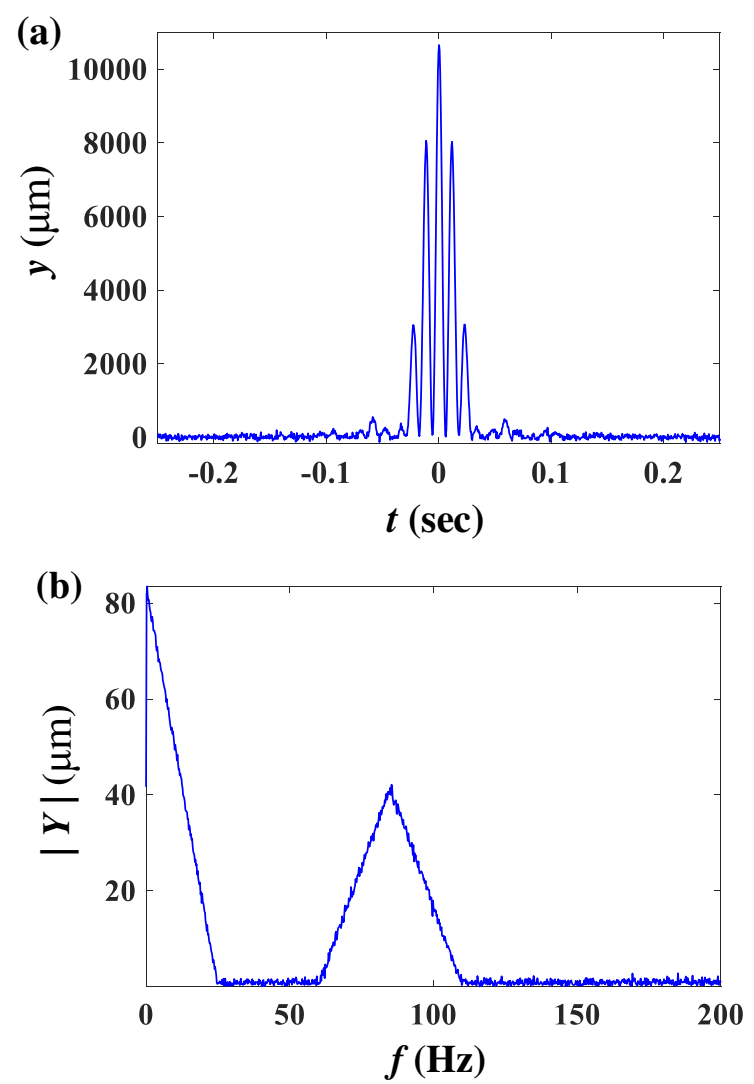

Figure 14 The time sequence and amplitude spectrum of the output of the second nonlinear system: (a) the time sequence, (b) the amplitude spectrum

Using the CSA method, the first five order NOFRFs $\left\{G_{i}(\mathrm{j} \omega), i=1, \cdots, 5\right\}$ of the second system are obtained and their amplitude spectra $\left\{\left|G_{i}(\mathrm{j} \omega)\right|\right\}$ are as shown in Figure 15, which reflect the amplitude-frequency response characteristics of each order nonlinearity of the system respecting to the input. Like the first nonlinear system simulated above, all the amplitude spectra of the second to fifth order NOFRFs have higher magnitudes in the frequency range of $30 \sim 55 \mathrm{~Hz}$ which is approximately equal to the original input frequency range. Besides, many distinct peaks exist in the lower and higher frequency ranges, e.g. $\left|G_{3}(\mathrm{j} \omega)\right|$ in the range of $0 \sim 20 \mathrm{~Hz}$, indicating that the NOFRFs also have notable gains on the input at the frequencies. However, because the $i$ th order output frequency response is equal to the product of $G_{i}(\mathrm{j} \omega)$ and $X_{i}(\mathrm{j} \omega)$ as given by Eq. (6), the output frequency responses 
of different orders depend not merely on the NOFRFs but also on the input.
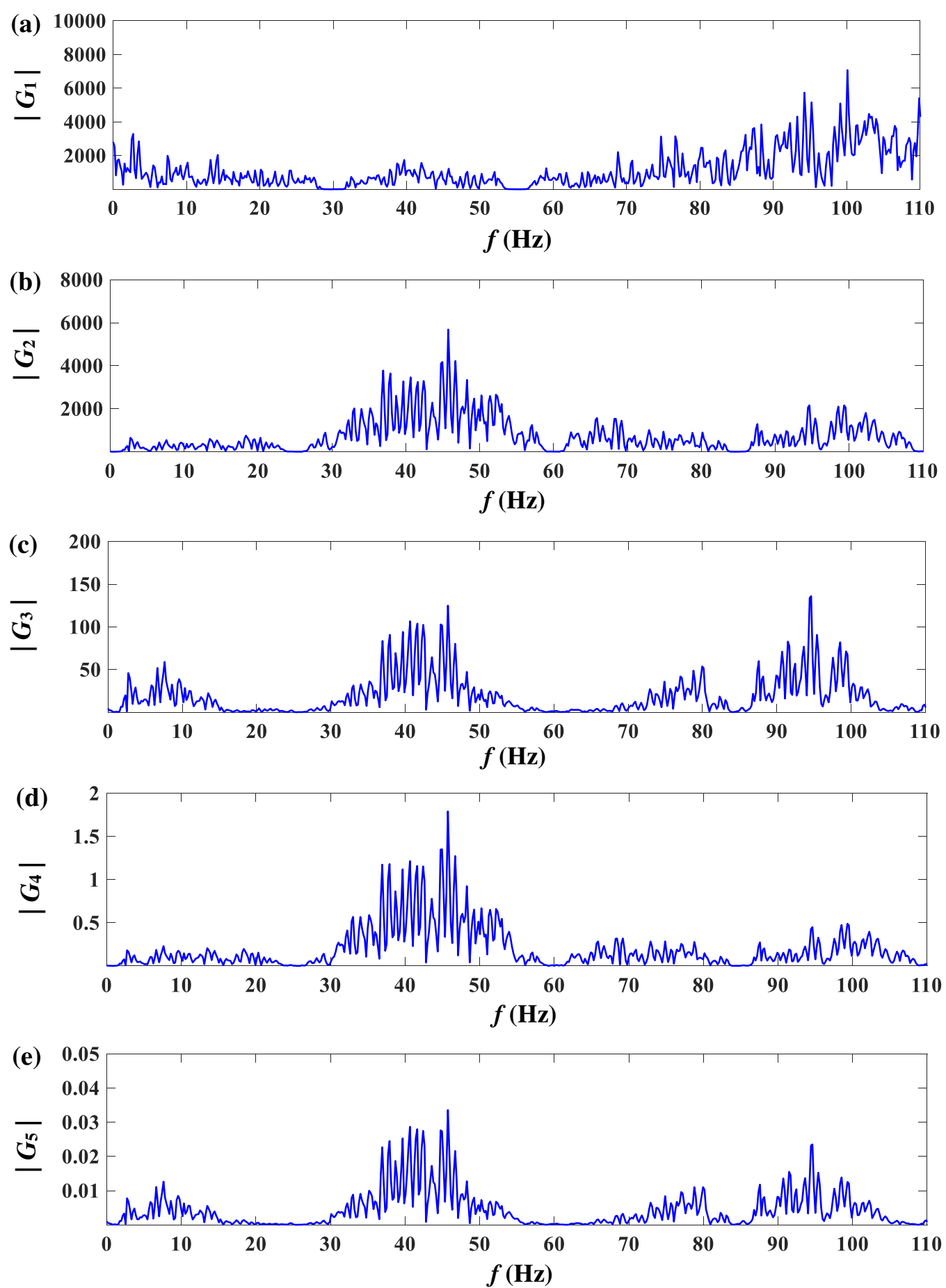

Figure 15 The amplitude spectra of the first five order NOFRFs of the second nonlinear system: (a) $\left|G_{1}(f)\right| ;(\mathrm{b})\left|G_{2}(f)\right| ;(\mathrm{c})\left|G_{3}(f)\right|$; (d) $\left|G_{4}(f)\right| ;(\mathrm{e})\left|G_{5}(f)\right|$

The magnitudes of the unconditioned output response spectra $\left\{Y_{i}(\mathrm{j} \omega), i=1, \cdots, 5\right\} \quad$ and the summation $\left|\sum_{i=1}^{5} Y_{i}(\mathrm{j} \omega)\right|$ are as plotted in Figure 16. Obviously, the second and fourth order inputs give rise to prominent output responses in the frequency ranges of $0 \sim 25 \mathrm{~Hz}$ and $60 \sim 110 \mathrm{~Hz}$. Although the first, third and fifth order inputs generate remarkable output responses in the frequency range of $30 \sim 55 \mathrm{~Hz}$, the amplitude of their summation 
decreases nearly to zero due to interferences, as shown in Figure 16(f). Similarly, in the higher frequency range of
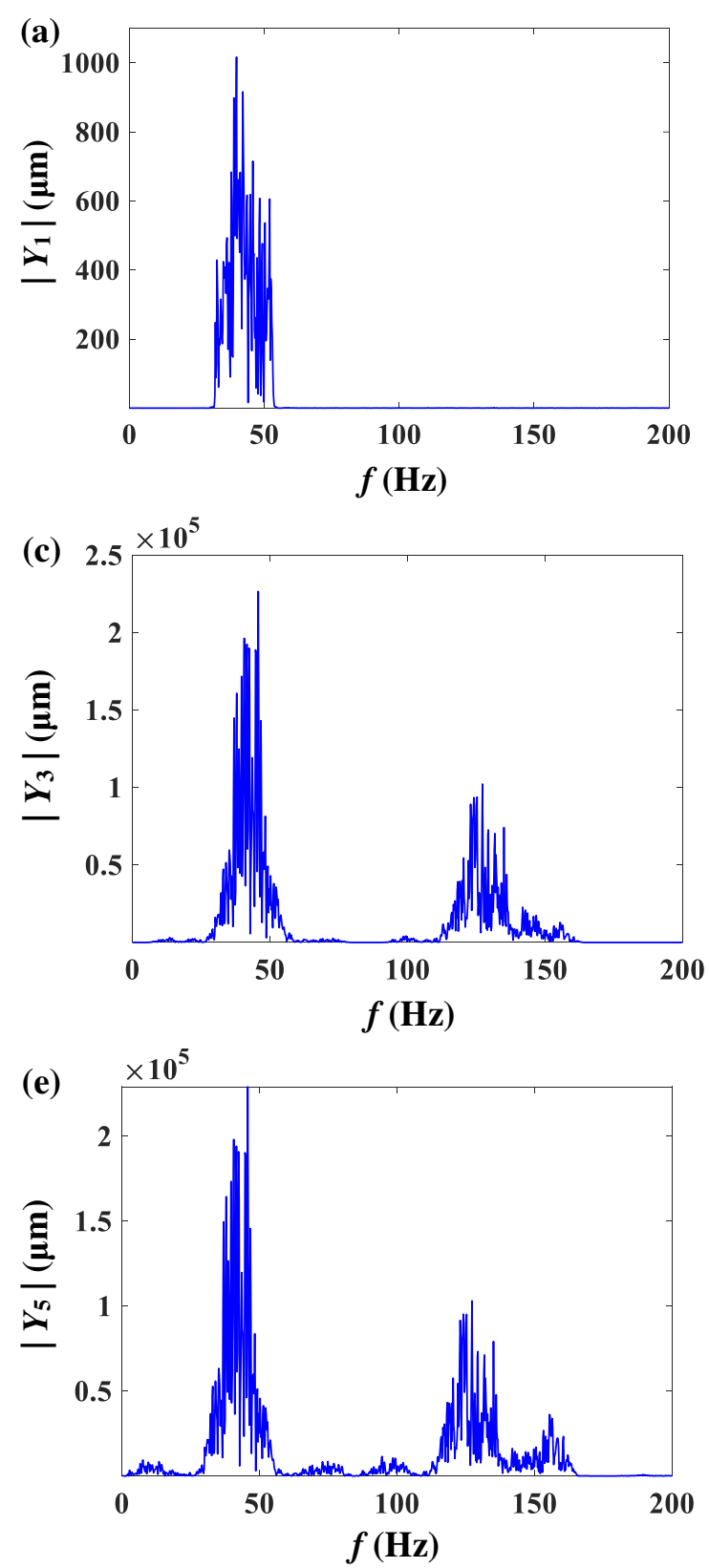

$110 \sim 170 \mathrm{~Hz}$, the output responses of the third to fifth orders are also reduced nearly to zero by interferences.
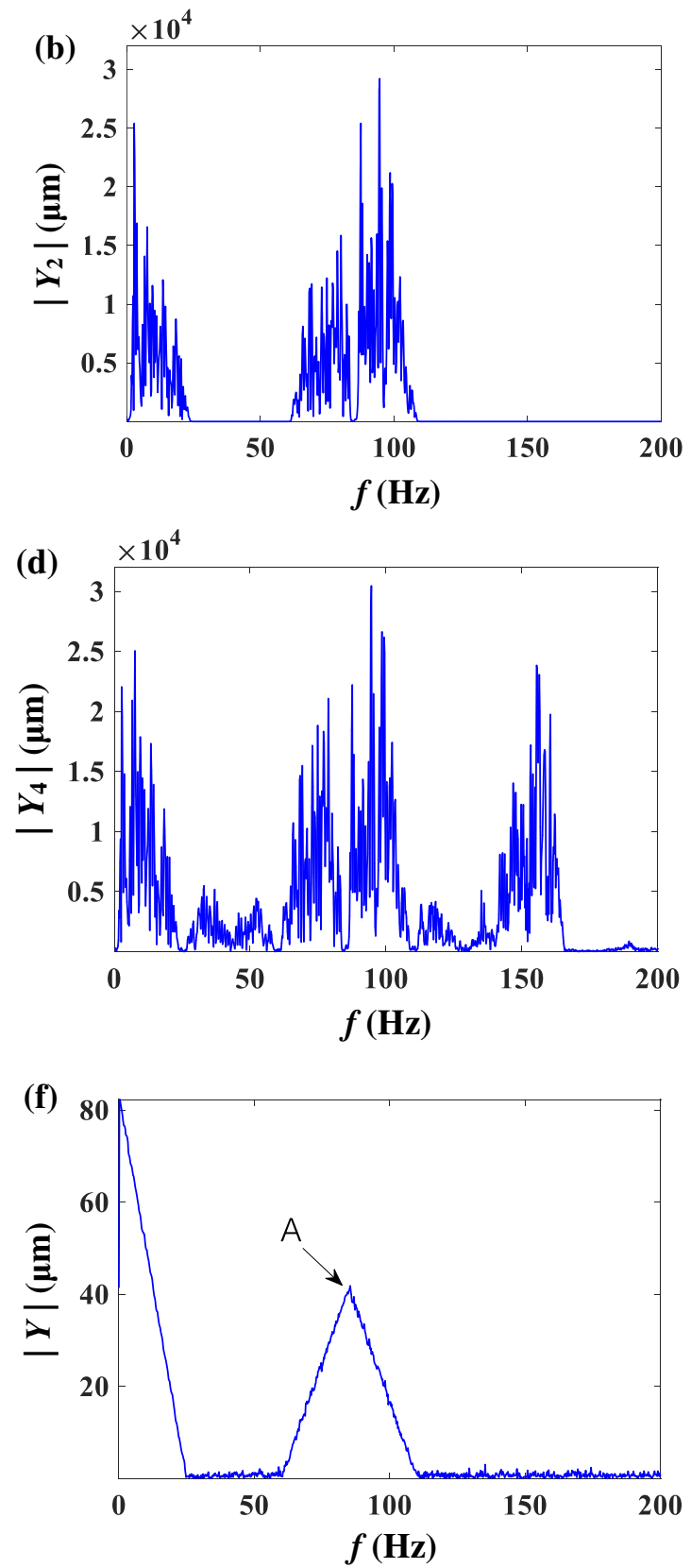

Figure 16 The amplitude spectra of the first five order unconditioned output components of the second nonlinear system and their summing spectrum: (a) $\left|Y_{1}(f)\right|$; (b) $\left|Y_{2}(f)\right| ;$ (c) $\left|Y_{3}(f)\right|$; (d) $\left|Y_{4}(f)\right| ;$ (e) $\left|Y_{5}(f)\right|$; (f) $|Y(f)|$

The summation of the unconditioned output response components is very close to the result of Runge-Kutta method shown in Figure 14(b), verifying numerically that the CSA based approach is effective in distinguishing the contributions of each order nonlinearity to the output of the system. For example, at the frequency of $85.23 \mathrm{~Hz}$ where the highest peak (i.e., the peak A) takes place in the range of $60 \sim 110 \mathrm{~Hz}$, the first five order output responses and their summation are listed in Table 1. The magnitudes of the five order response components equal to $0.5021 \mu \mathrm{m}$, $241.4586 \mu \mathrm{m}, \quad 9.8617 \times 10^{-5} \mu \mathrm{m}, 488.2731 \mu \mathrm{m}$ and $289.0718 \mu \mathrm{m}$, respectively. Even though the magnitudes of the second, fourth and fifth order responses reach up to several hundred at the frequency, the total output 
magnitude reduces to $41.4629 \mu \mathrm{m}$ as a result of interferences. In addition, it is uncovered that the contribution of the first order is much smaller than the second, fourth and fifth orders at the frequency, and the contribution of the third order is even slighter. The total output magnitude at the frequency is approximately equal to the numerical result $40.7236 \mu \mathrm{m}$ obtained by the fourth order Runge-Kutta method with a deviation of $1.815 \%$ which is mainly due to the noise $n_{y}(t)$ acting on the output.

Table 2 The contributions of the first five order nonlinearities to the output of the second nonlinear system and their summation at the frequency of $85.23 \mathrm{~Hz}$.

\begin{tabular}{ll}
\hline \multicolumn{1}{c}{ Items } & \multicolumn{1}{c}{ Values } \\
\hline$Y_{1}(f)$ & $0.2925-0.4081 \mathrm{j}$ \\
$Y_{2}(f)$ & $1.2291 \times 10^{2}-2.0784 \times 10^{2} \mathrm{j}$ \\
$Y_{3}(f)$ & $-5.3646 \times 10^{-5}+8.2749 \times 10^{-5} \mathrm{j}$ \\
$Y_{4}(f)$ & $-2.1703 \times 10^{2}+4.3739 \times 10^{2} \mathrm{j}$ \\
$Y_{5}(f)$ & $1.0915 \times 10^{2}-2.6767 \times 10^{2} \mathrm{j}$ \\
$\sum_{n=1}^{5} Y_{n}(f)$ & $15.3246-38.5271 \mathrm{j}$ \\
$\left|\sum_{n=1}^{5} Y_{n}(f)\right|$ & 41.4629 \\
\hline
\end{tabular}

\section{Conclusions}

(1) In this study, a digital method of evaluating NOFRFs of the nonlinear systems with a general input is proposed by applying CSA technique, i.e., the NOFRFs-CSA method as shown in Figure 3.

(2) The interferences among various order output frequency components are evaluated and removed in the analysis procedure, and the NOFRFs are identified consequently.

(3) Based on the NOFRFs-CSA method, the contributions of different order nonlinearities of the nonlinear system to the output can be distinguished in frequency domain, revealing the nonlinear frequency response characteristics of the nonlinear system.

(4) Two kinds of nonlinear systems expressed by Eq. (23) and Eq. (25) were simulated with a same general input respectively. The results reached by the proposed method are very close to the numerical results obtained by the fourth order Runge-Kutta method, verifying the effectiveness of the proposed method on the evaluation of NOFRFs.

\section{Declaration}

\section{Acknowledgements}

Not applicable.

\section{Funding}

Supported by National Natural Science Foundation of China (Grant No. 51605190) and National Key Research and Development Program of China (Grant No. 2019YFB1707301).

\section{Availability of data and materials}

The datasets supporting the conclusions of this article are included within the article.

\section{Authors' contributions}

The author' contributions are as follows: WTL and YZ were in charge of the whole algorithm deduction; WTL wrote the manuscript; SRW assisted with simulation studies and numerical calculations.

\section{Competing interests}

The authors declare no competing financial interests.

\section{Consent for publication \\ Not applicable}

\section{Ethics approval and consent to participate}

Not applicable

\section{References}

[1] V Volterra. Theory of functionals and of integral and integrodifferential equations. New York: Dover Publications, 2005.

[2] J C Peyton Jones, S A Billings, Interpretation of non-linear frequency response functions. International Journal of Control, 1990, 52(2): 319-346.

[3] B Zhang, S A Billings. Volterra series truncation and kernel estimation of nonlinear systems in the frequency domain. Mechanical Systems and Signal Processing, 2017, 84, pp. 39-57.

[4] Y C Kim, W F Wong, E J Powers, et al. Extension of the coherence function to quadratic models. Proceedings of the IEEE, 1979, 67(3): 428-429.

[5] J Y Hong, Y C Kim, E J Powers. On modeling the nonlinear relationship between fluctuations with Nonlinear transfer functions. Proceedings of the IEEE, 1980, 68(8): 1026-1027.

[6] W T Liu, Y Zhang, Z J Feng, et al. A study on waviness induced vibration of ball bearings based on signal coherence theory. Journal of Sound and Vibration, 2014, 333: 6107-6120.

[7] Y C Kim, E J Powers. A digital method of modeling quadratically nonlinear systems with a general random input. IEEE Transactions on Acoustics, Speech, and Signal Processing, 1988, 36(11): 1758-1769.

[8] Y S Cho, S B Kim, E L Hixson, et al. A digital technique to estimate second-order distortion using higher order coherence spectra. IEEE Transactions on Signal Processing, 1992, 40(5): 1029-1040. 
[9] C K An, E J Powers, C P Ritz. A digital method of modeling two-input quadratic systems with general random inputs. IEEE Transactions on Signal Processing, 1991, 39(10): 2320-2323.

[10]J C Peyton Jones. Simplified computation of the Volterra frequency response functions of non-linear systems. Mechanical Systems and Signal Processing, 2007, 21: 1452-1468.

[11]Z Q Lang, S A Billings. Output frequency characteristics of nonlinear systems. International Journal of Control, 1996, 64(6): 1049-1067.

[12]J C Peyton Jones, K Choudhary. Output frequency response characteristics of nonlinear systems, part I: general multi-tone inputs. International Journal of Control, 2012, 85(9): 1263-1278.

[13]O Scussel, S Silva da. Output-only identification of nonlinear systems via Volterra series. ASME Journal of Vibration and Acoustics, 2016, 138(4): 041012.

[14]R M Lin, T Y Ng. Higher-order FRFs and their applications to the identifications of continuous structural systems with discrete localized nonlinearities. Mechanical Systems and Signal Processing, 2018, 108: 326-346.

[15]P Marzocca, J M Nichols, A Milanese, et al. Second-order spectra for quadratic nonlinear systems by Volterra functional series: analytical description and numerical simulation. Mechanical Systems and Signal Processing, 2008, 22: 1882-1895.

[16]A Chatterjee, N S Vyas. Non-linear parameter estimation with Volterra series using the method of recursive iteration through harmonic probing. Journal of Sound and Vibration, 2003, 268: 657-678.

[17]O Scussel, S Silva da. The harmonic probing method for output-only nonlinear mechanical systems. Journal of the Brazilian Society of Mechanical Sciences and Engineering, 2017, 39(9): 3329-3341.

[18]A Chatterjee. Identification and parameter estimation of a bilinear oscillator using Volterra series with harmonic probing. International Journal of Non-Linear Mechanics, 2010, 45: 12-20.

[19]G M Lee. Estimation of Non-linear system parameters using higher-order frequency response functions. Mechanical Systems and Signal Processing, 1997, 11(2): 219-228.

[20]A Chatterjee. Structural damage assessment in a cantilever beam with a breathing crack using higher order frequency response functions. Journal of Sound and Vibration, 2010, 329: 3325-3334.

[21]A Chatterjee, N S Vyas. Convergence analysis of Volterra series response of nonlinear systems subjected to harmonic excitation. Journal of Sound and vibration, 2000, 236(2): 339-358.

[22]Z K Peng, Z Q Lang. On the convergence of the Volterra-series representation of the duffing's oscillators subjected to harmonic excitations. Journal of Sound and Vibration, 2007, 305: 322-332.

[23]Z Q Lang, S A Billings. Energy transfer properties of non-linear systems in the frequency domain. International Journal of Control, 2005, 78(5): 345-362.

[24]Z K Peng, Z Q Lang, S A Billings, et al. Analysis of bilinear oscillators under harmonic loading using nonlinear output frequency response functions. International Journal of Mechanical Sciences, 2007, 49(11): 1213-1225.

[25]Z K Peng, Z Q Lang, S A Billings, et al. Comparisons between harmonic balance and nonlinear output frequency response function in nonlinear system analysis. Journal of Sound and Vibration, 2008, 311: 56-73.

[26]Z K Peng, Z Q Lang, S A Billings. Analysis of locally nonlinear MDOF systems using nonlinear output frequency response functions. ASME Journal of Vibration and Acoustics, 2009, 131(5): 051007.

[27]Z Q Lang, G Park, C R Farrar, et al. Transmissibility of non-linear output frequency response functions with application in detection and location of damage in MDOF structural systems. International Journal of Non-Linear Mechanics, 2011, 46(6): 841-853.

[28]J S Bendat. System identification from multiple input/output data. Journal of Sound and Vibration, 1976, 49(3): 293-308.

\section{Biographical notes}

Wen-Tao Liu, born in 1982, is currently a lecturer at School of Mechanical Engineering, University of Jinan, China. He received his $\mathrm{PhD}$ degree from Tsinghua University, China, in 2016. His research interests include diagnoses of mechanical failure and intelligent manufacturing technologies.

Tel: +86-531-82765476; E-mail: me_liuwt@ @ujn.edu.cn

Yun Zhang, born in 1974, is currently an associate research fellow at Beijing Key Lab of Precision/Ultra-precision Manufacturing Equipment and Control, Tsinghua University, China. He received his PhD degree in Tsinghua University, China, in 2004. His research interests include precision or ultra-precision machining, intelligent manufacturing and optical detective technologies.

Tel: +86-010-62773264; E-mail: zhangyun@ mail.tsinghua.edu.cn

Shou-Ren Wang, born in 1966, is currently a professor at School of Mechanical Engineering, University of Jinan, China. He received his $\mathrm{PhD}$ degree from Shandong University, China, in 1996. His research interests include intelligent manufacturing processes and equipment.

Tel: +86-531-87109072; E-mail: me_wangsr@ujn.edu.cn 
Figures

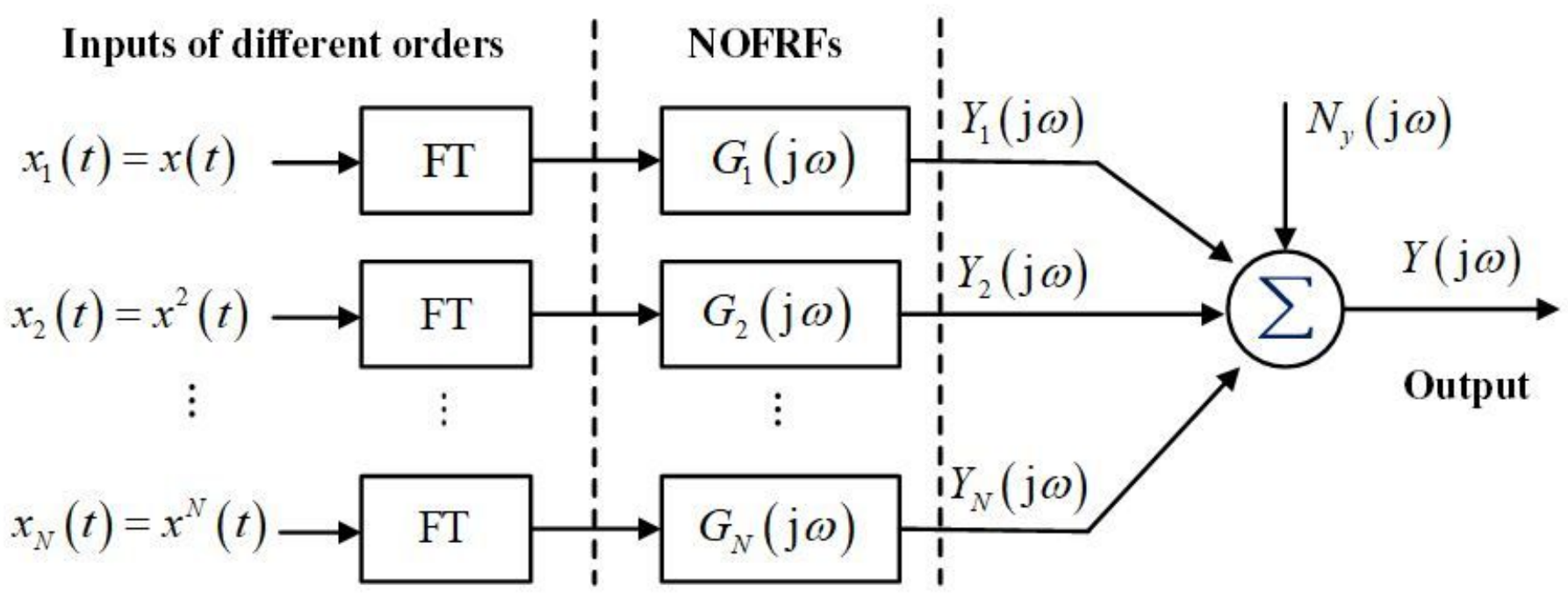

Figure 1

The MISO model of the nonlinear system linearized by NOFRFs

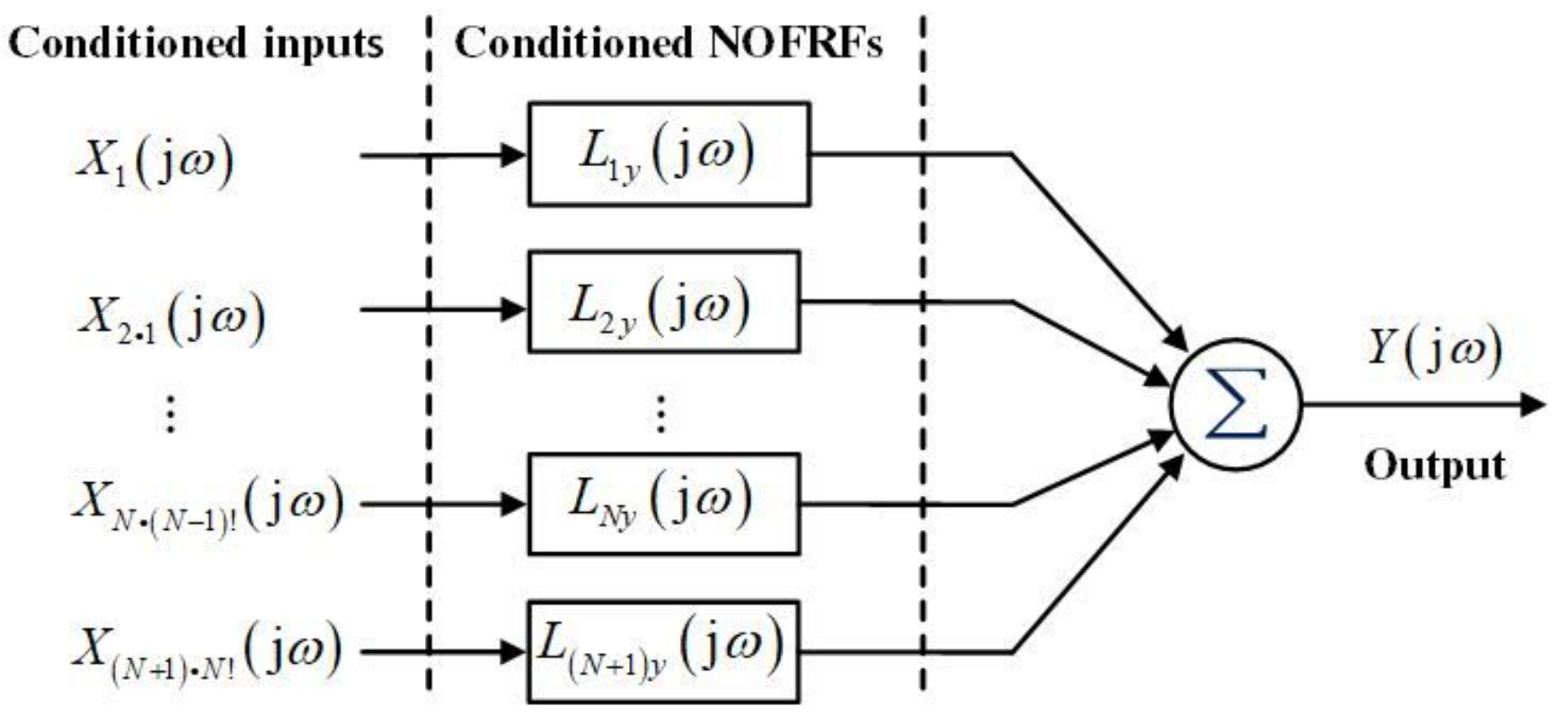

Figure 2

The CSA model of the nonlinear system linearized by NOFRFs. 
To decompose the nonlinear system under study into a MISO linear system with a series of power characterized inputs, i.e. $\left\{x^{n}(t), n=1,2, \cdots, N\right\}$.

To estimate the auto-power spectral densities of each order input and the measured output, i.e. $\left\{S_{i i}(\mathrm{j} \omega), i=1,2, \cdots, N\right\}$ and $S_{y y}(\mathrm{j} \omega)$, the cross-power spectral densities of different order inputs, i.e. $\left\{S_{i j}(\mathrm{j} \omega), i=1,2, \cdots, N, i<j \leq N\right\}$, and the cross-power spectral densities of each order input and the output, i.e. $\left\{S_{i y}(\mathrm{j} \omega), i=1,2, \cdots, N\right\}$.

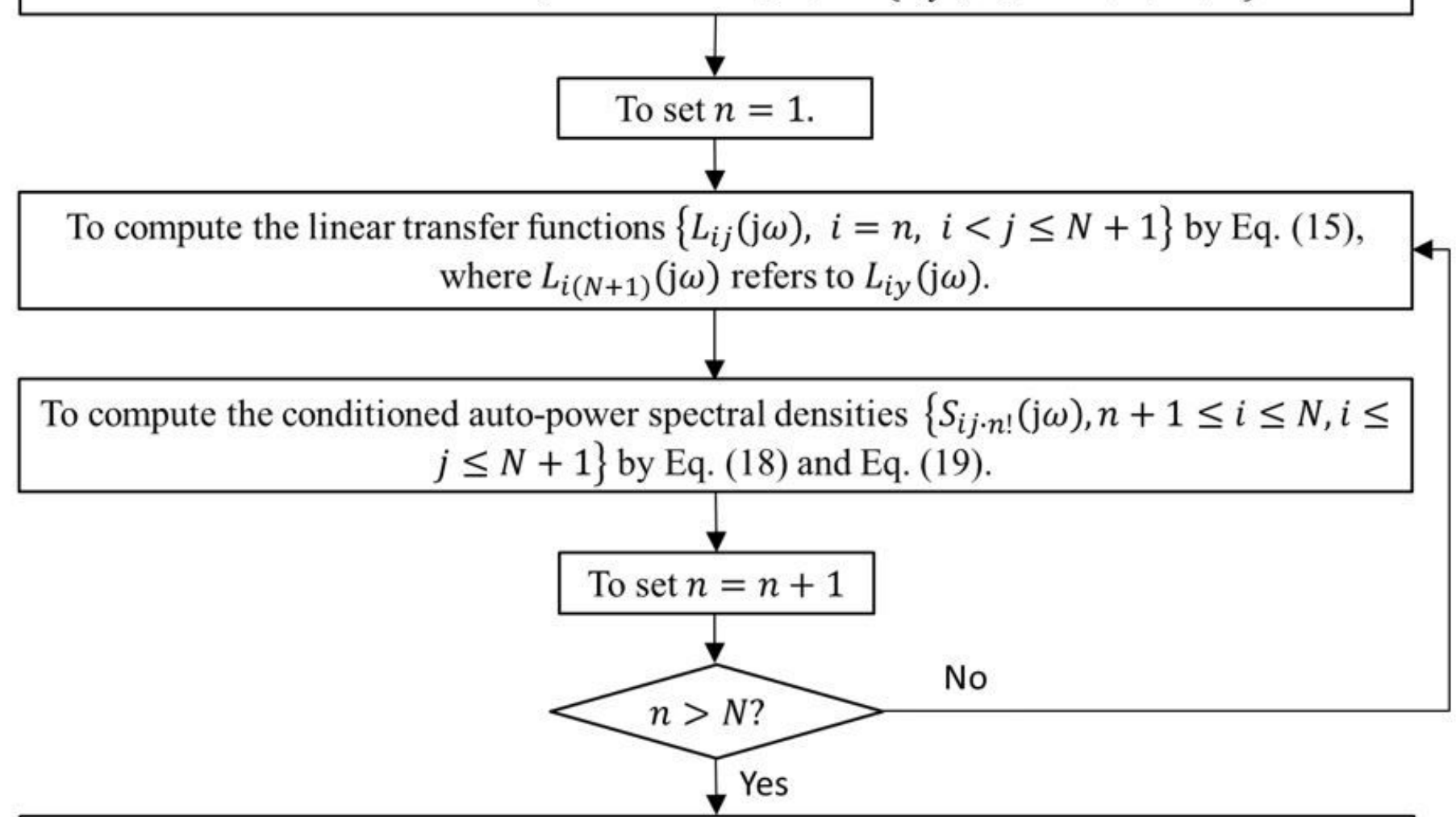

Obtain the NOFRFs $\left\{G_{i}(\mathrm{j} \omega), 1 \leq i \leq N\right\}$ of the nonlinear system by Eq. (20).

Figure 3

The proposed algorithm of evaluating NOFRFs based on CSA. 


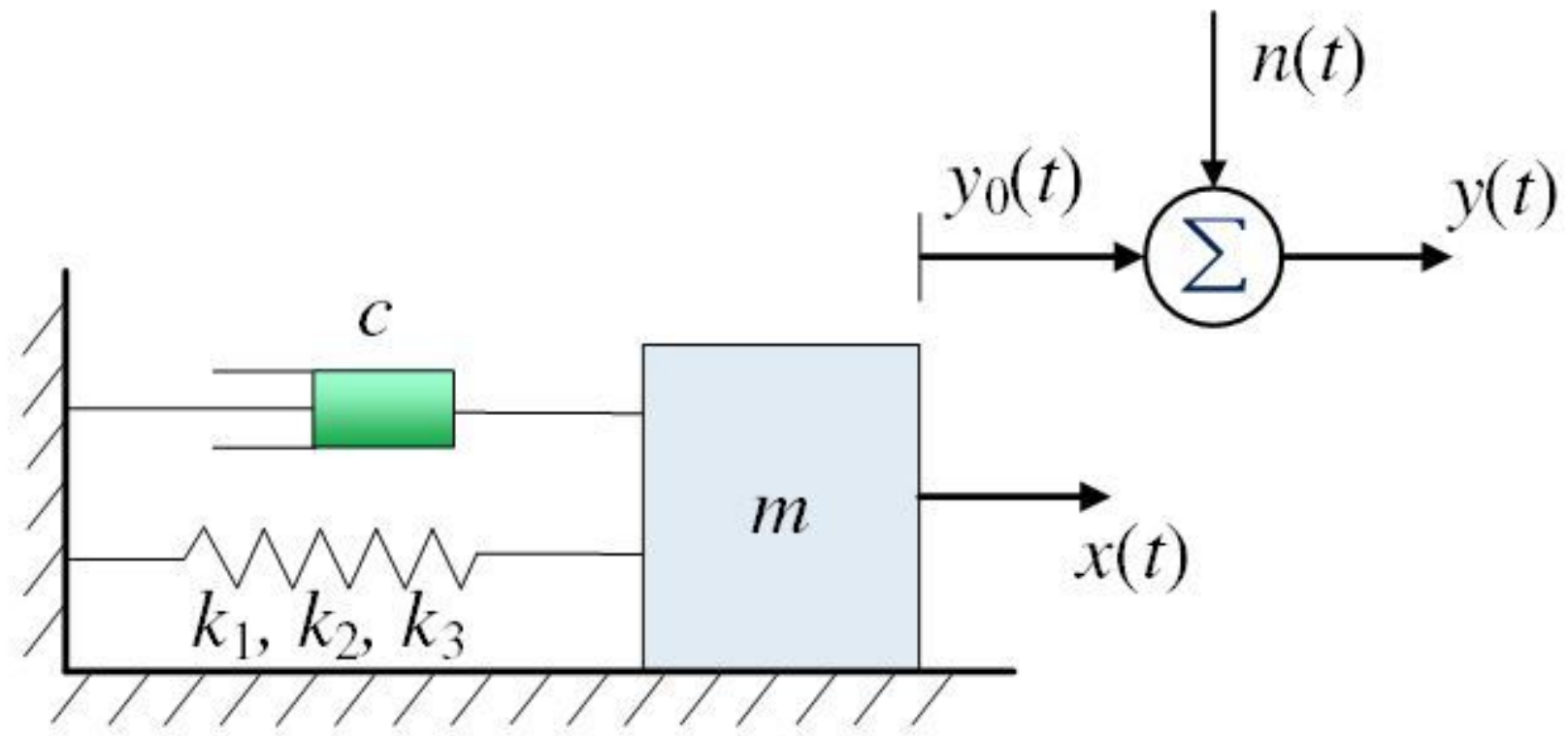

Figure 4

The first nonlinear system for simulation
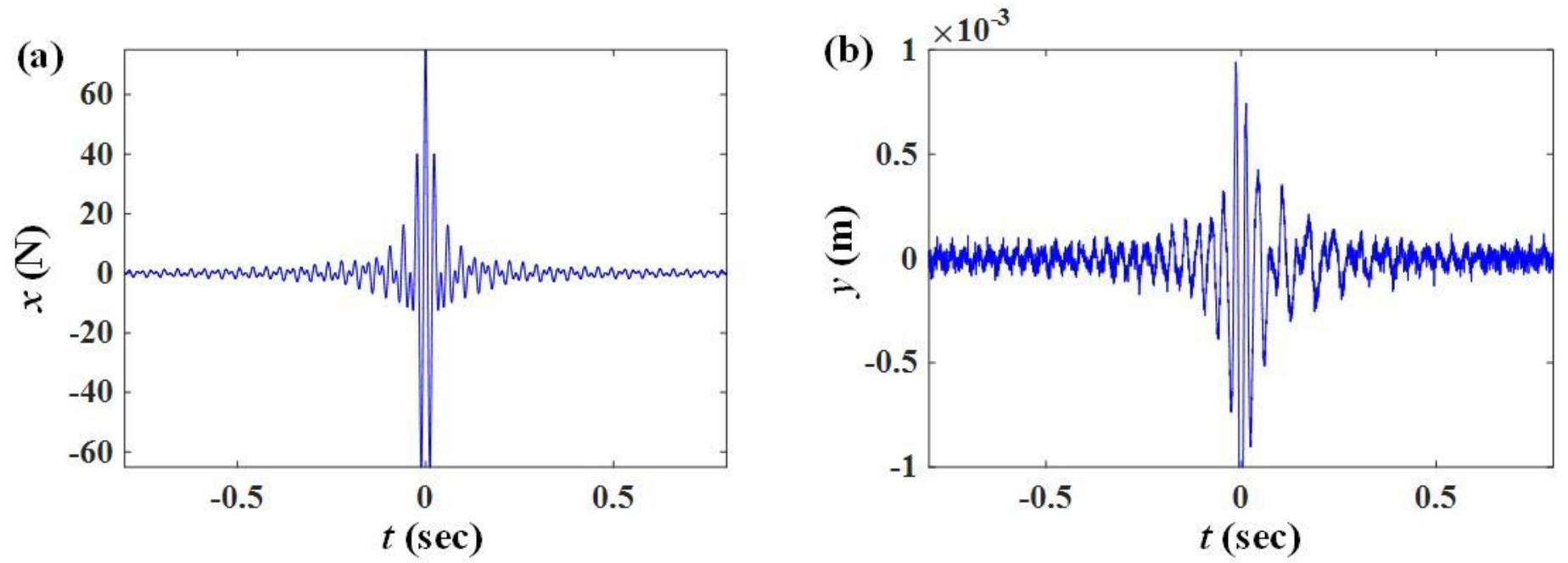

Figure 5

The time sequences of the system input and output: (a) the input, (b) the output. 

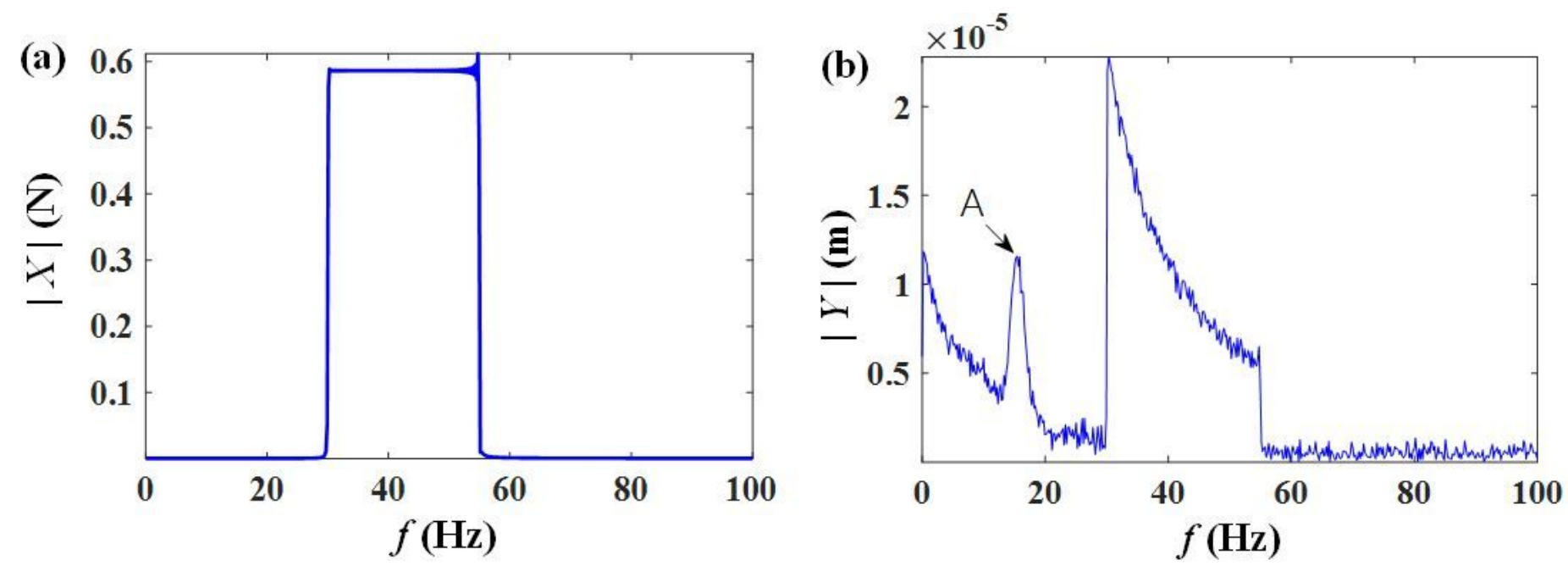

Figure 6

The amplitude spectra of the system input and output obtained by FFT: (a) the input, (b) the output.

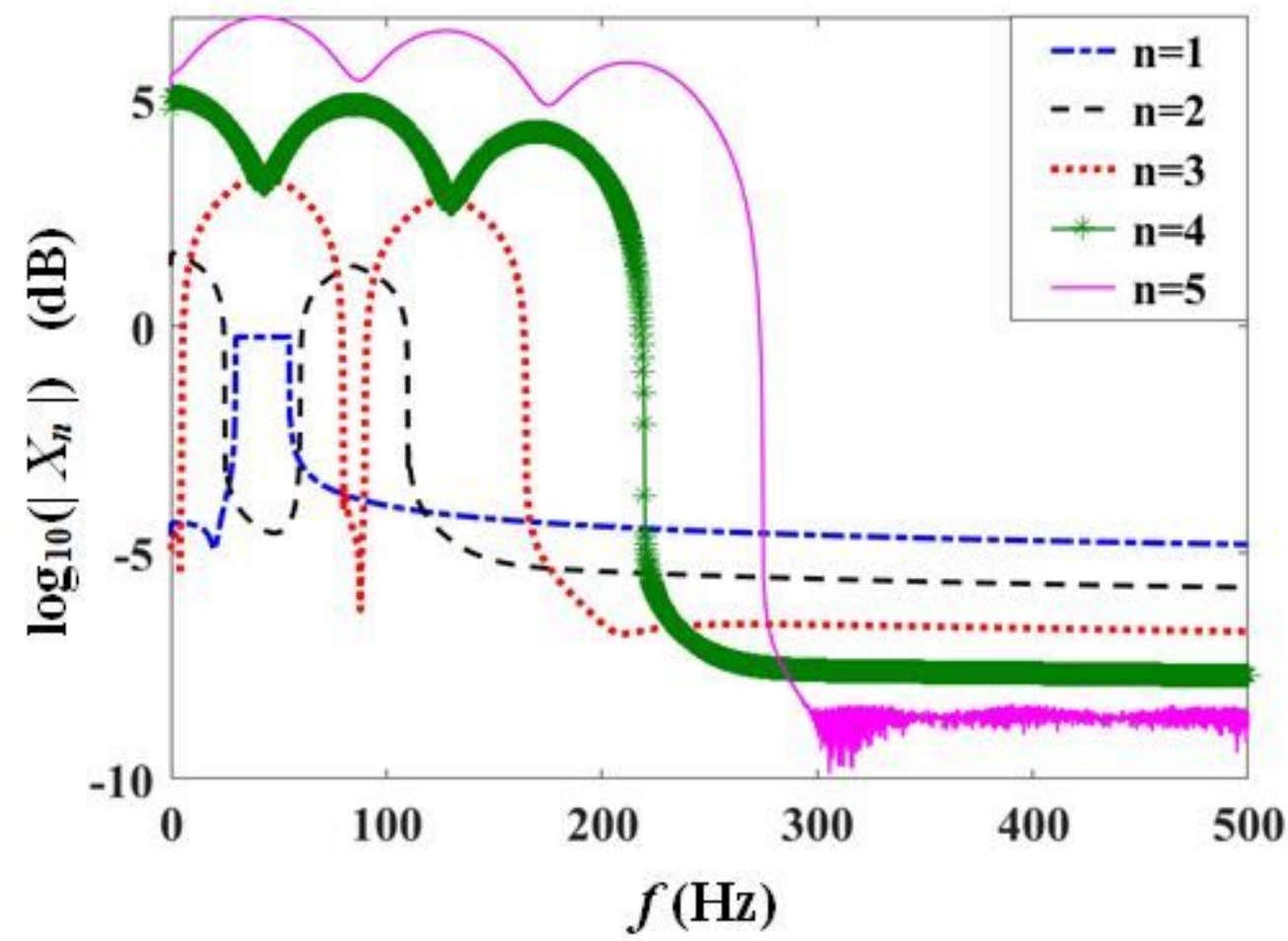

Figure 7

The amplitude spectra of the first five order inputs obtained by FFT 

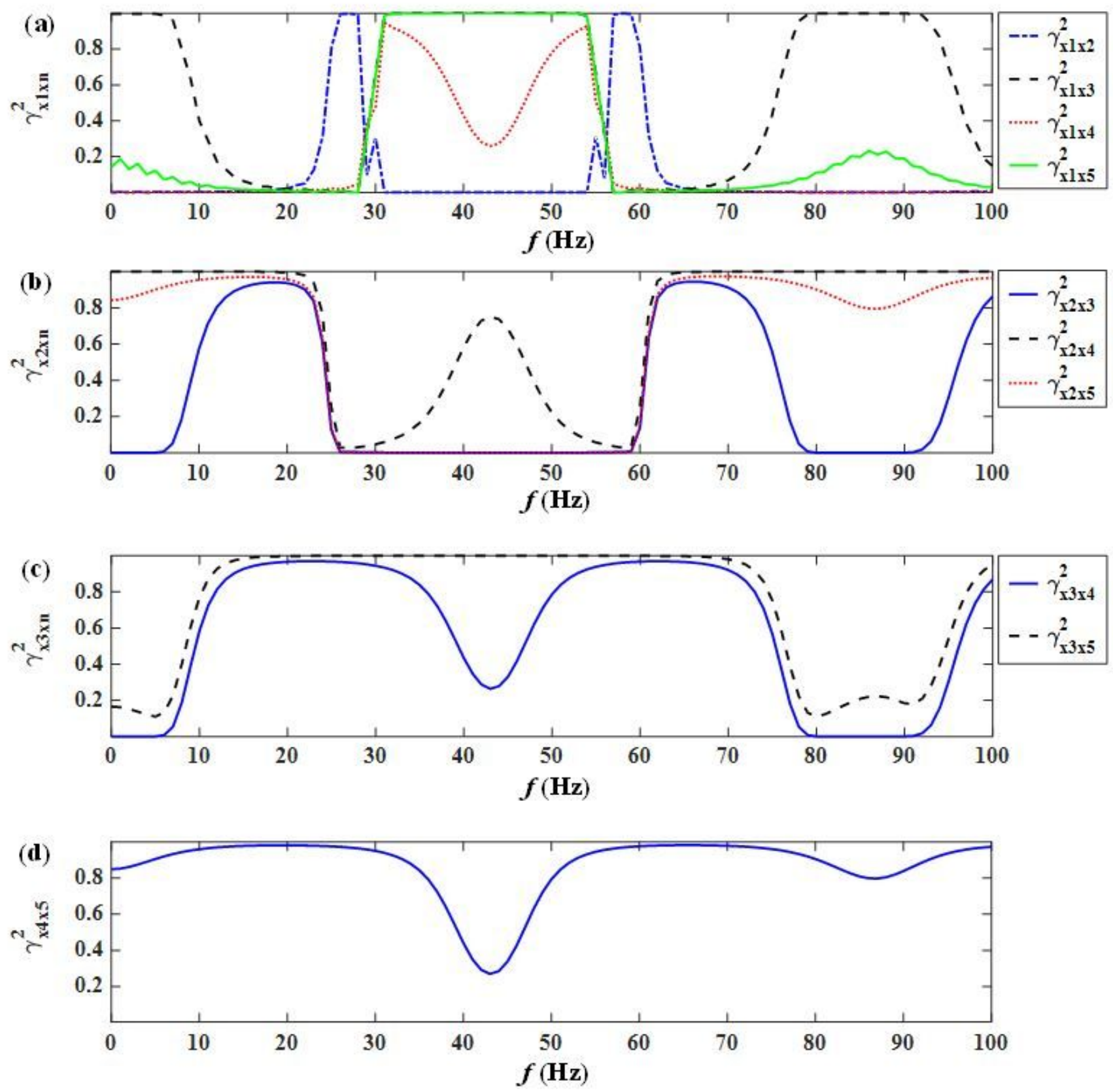

Figure 8

The coherence spectra of the input between different orders: (a) between the first and the higher orders, (b) between the second and the higher orders, (c) between the third and the higher orders, (d) between the fourth and the fifth orders. 


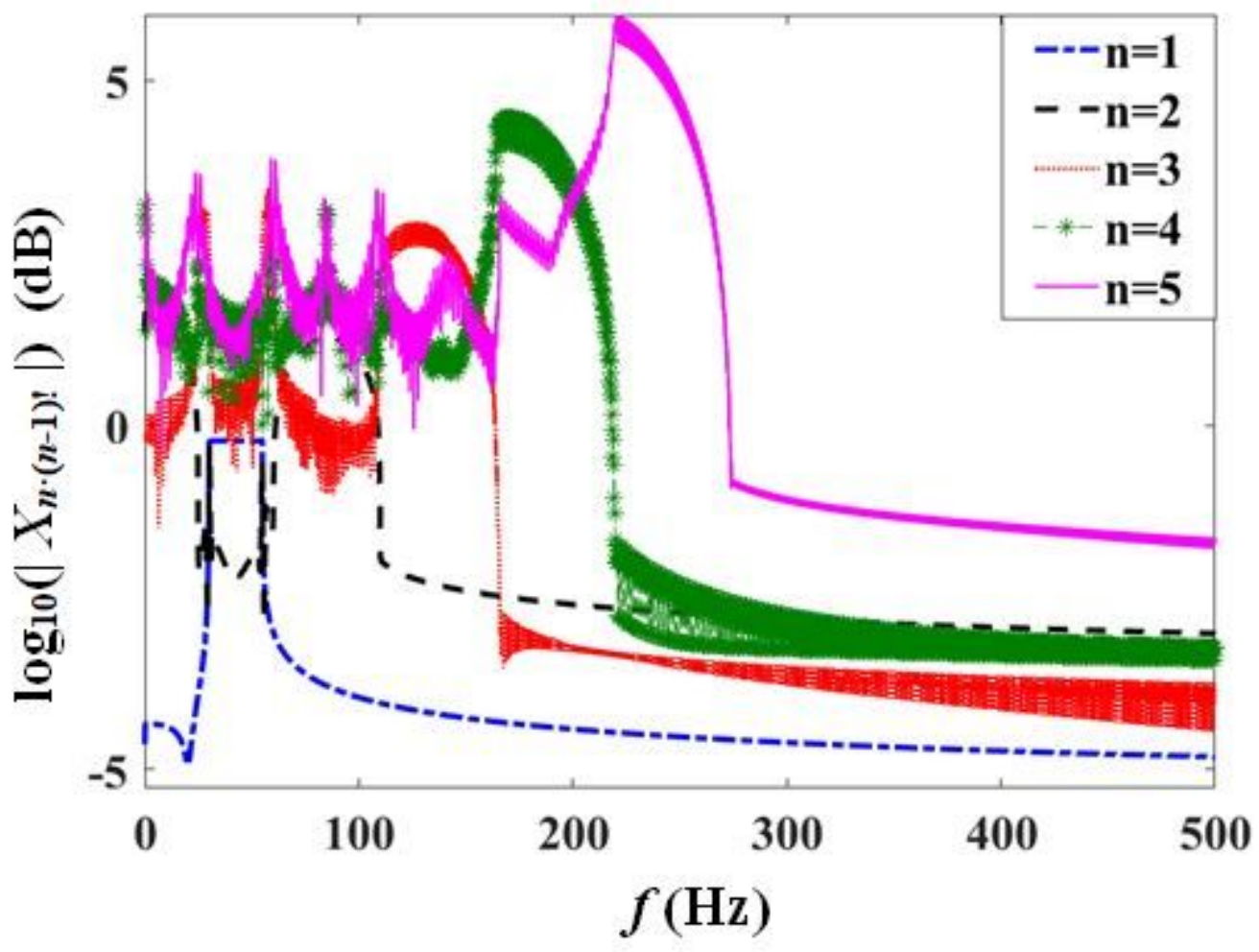

Figure 9

The amplitude spectra of the conditioned inputs 

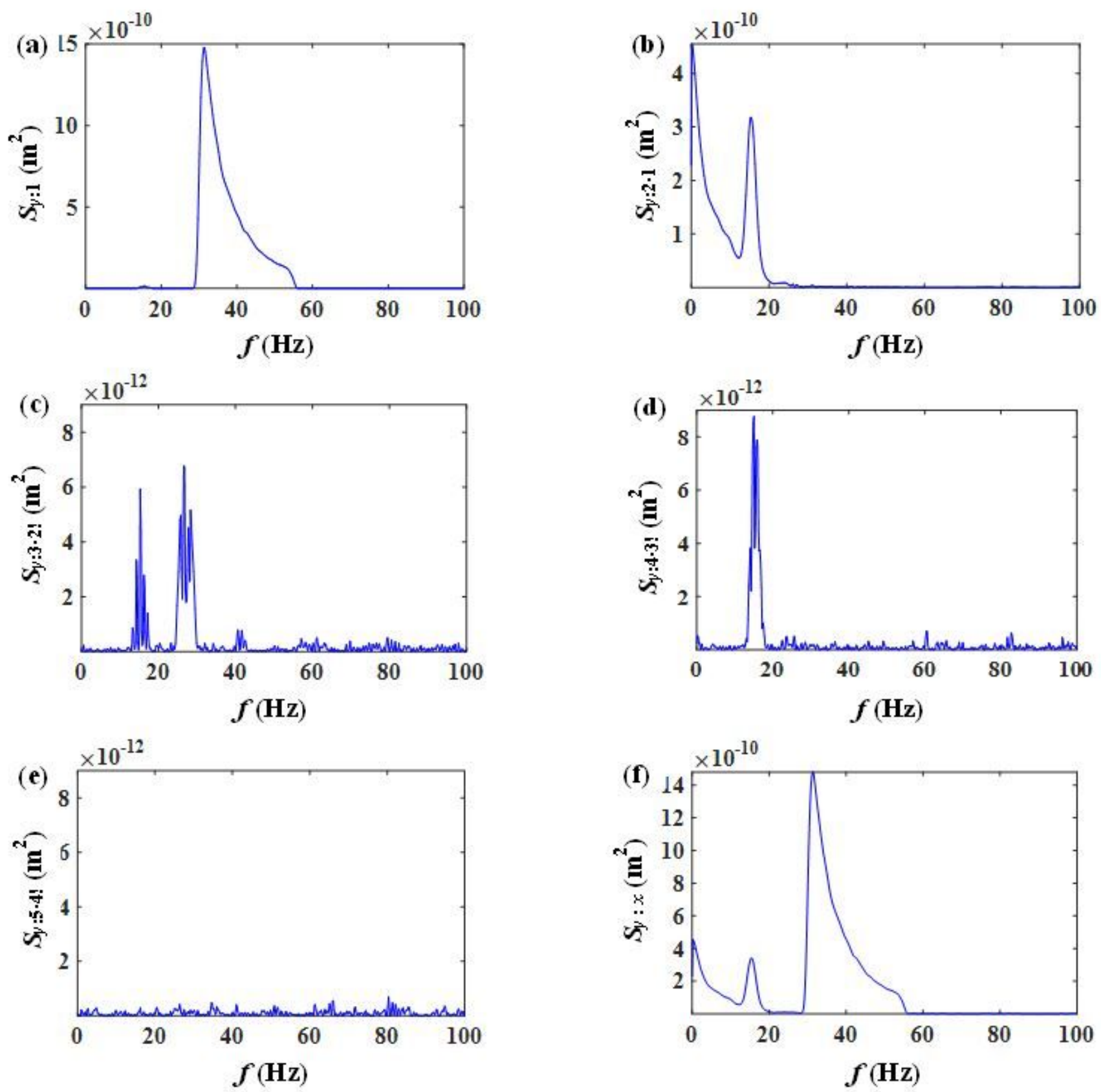

Figure 10

he power spectra of the first five conditioned outputs and their summation of the first nonlinear system:

(a) S_(y:1) (f), (b) S_(y:2 1) (f), (c) S_(y:3 2!) (f), (d) S_(y:4 3!) (f), (e) S_(y:5 4!) (f), (f) S_(y:x) (f). 

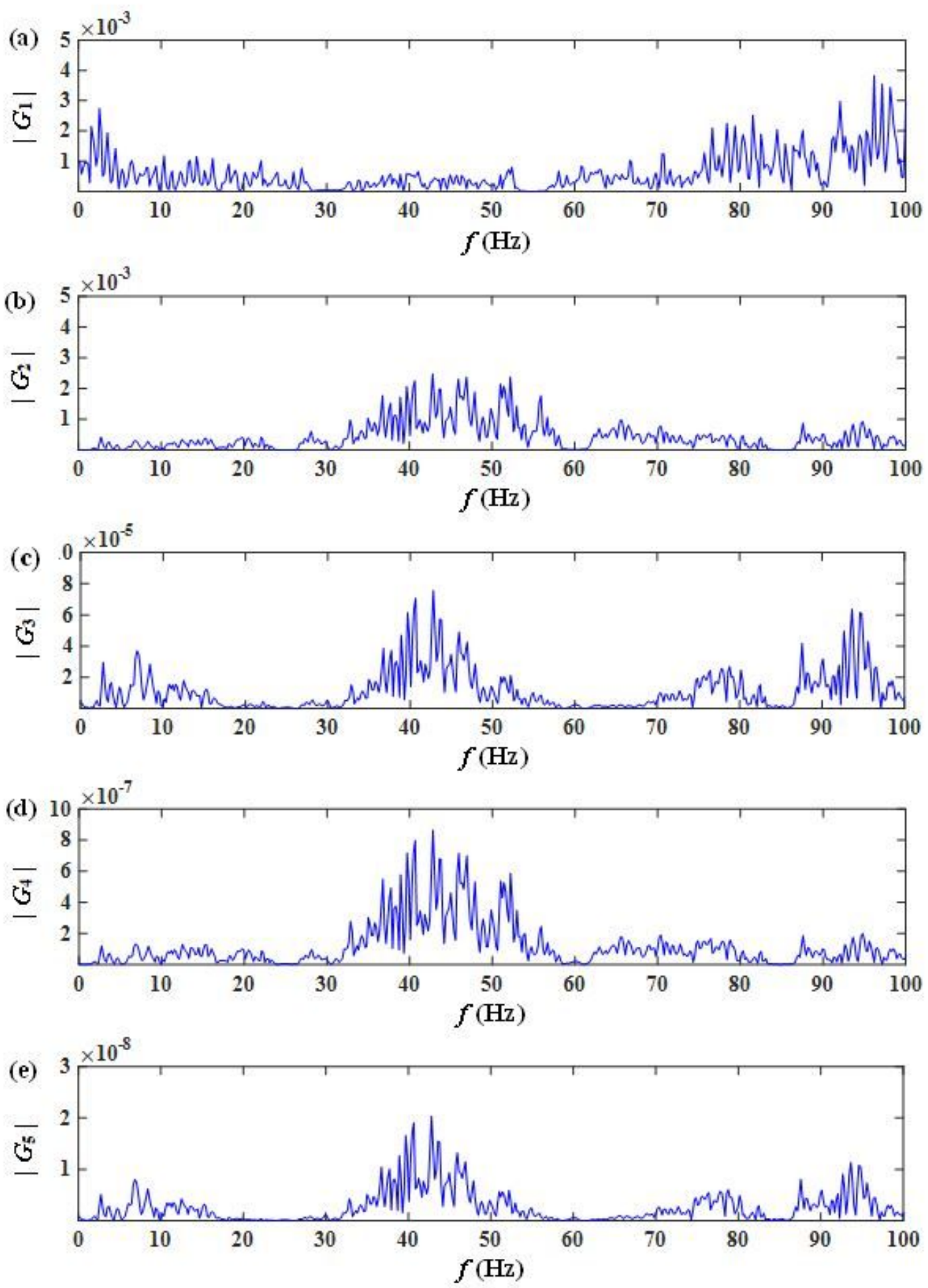

\section{Figure 11}

The amplitude spectra of the first five order NOFRFs of the first nonlinear system: (a) $\mid G_{-} 1$ (f)|; (b) |G_2 (f)|; (c) $\left|G \_3 ~(f)\right| ;(d)\left|G \_4 ~(f)\right| ;(e)\left|G \_5 ~(f)\right|$. 

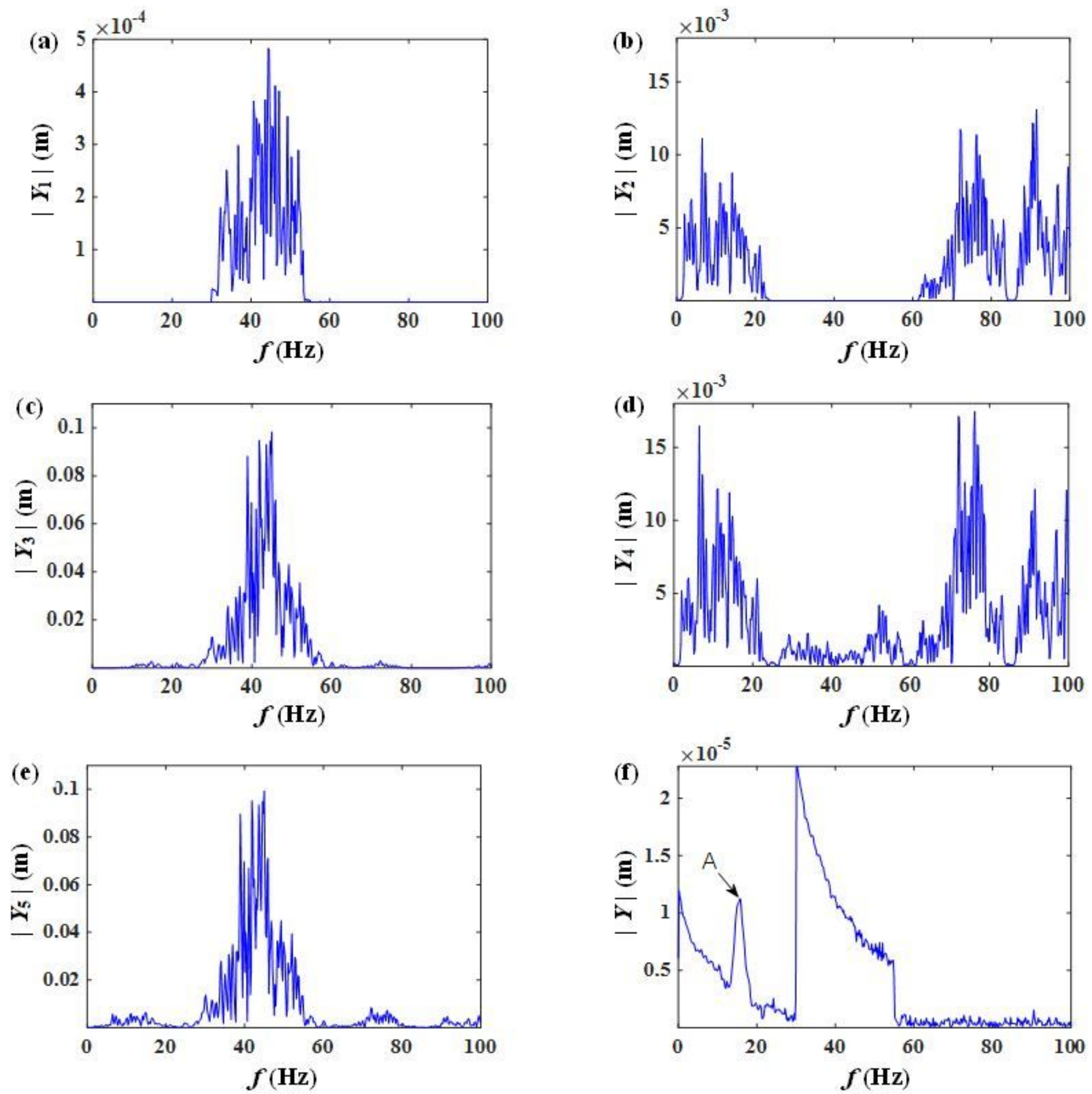

Figure 12

The amplitude spectra of the first five order unconditioned output components of the first nonlinear system and the summing spectrum of them: (a) |Y_1 (f)|; (b) $\mid Y \_2$ (f)|; (c) $\left|Y \_3 ~(f)\right| ;$ (d) $\mid Y \_4$ (f)|; (e) |Y_5 (f)|; (f) $|Y(f)|$. 


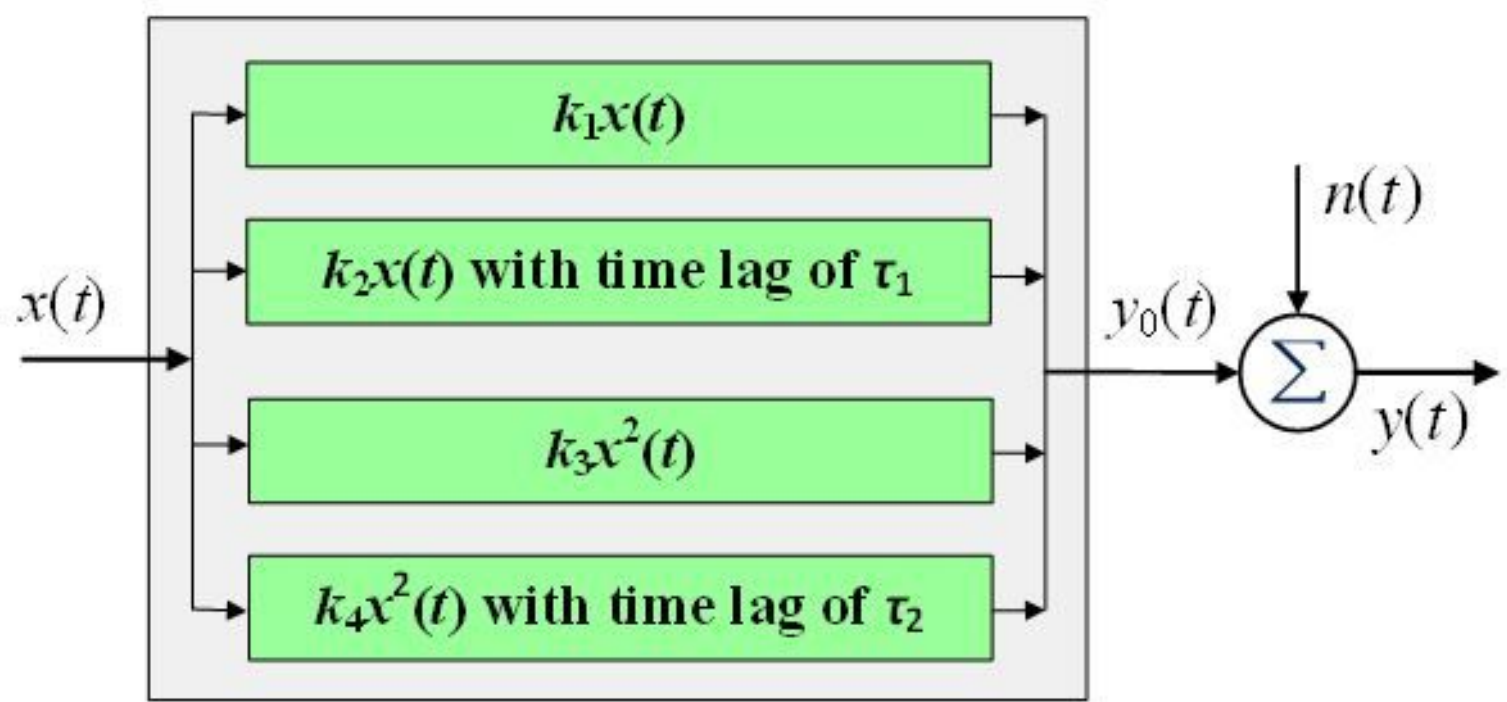

Figure 13

The quadratic nonlinear system with time lags for simulation
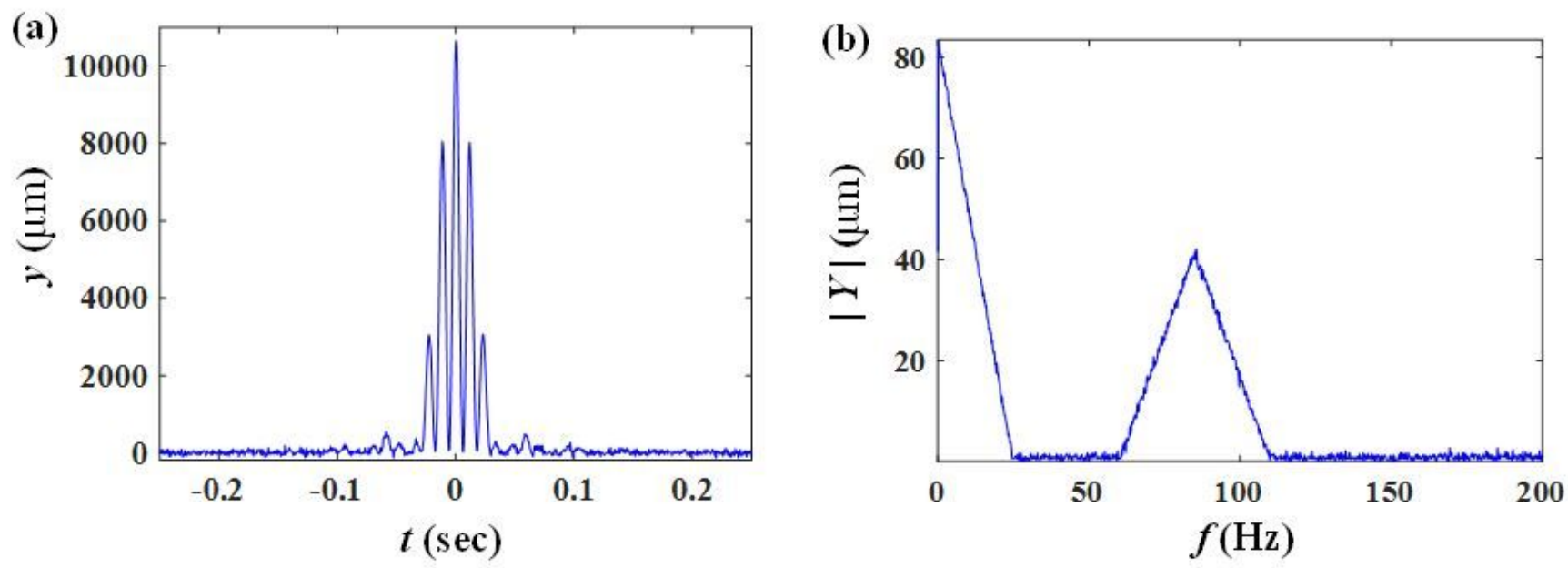

Figure 14

The time sequence and amplitude spectrum of the output of the second nonlinear system: (a) the time sequence, (b) the amplitude spectrum 

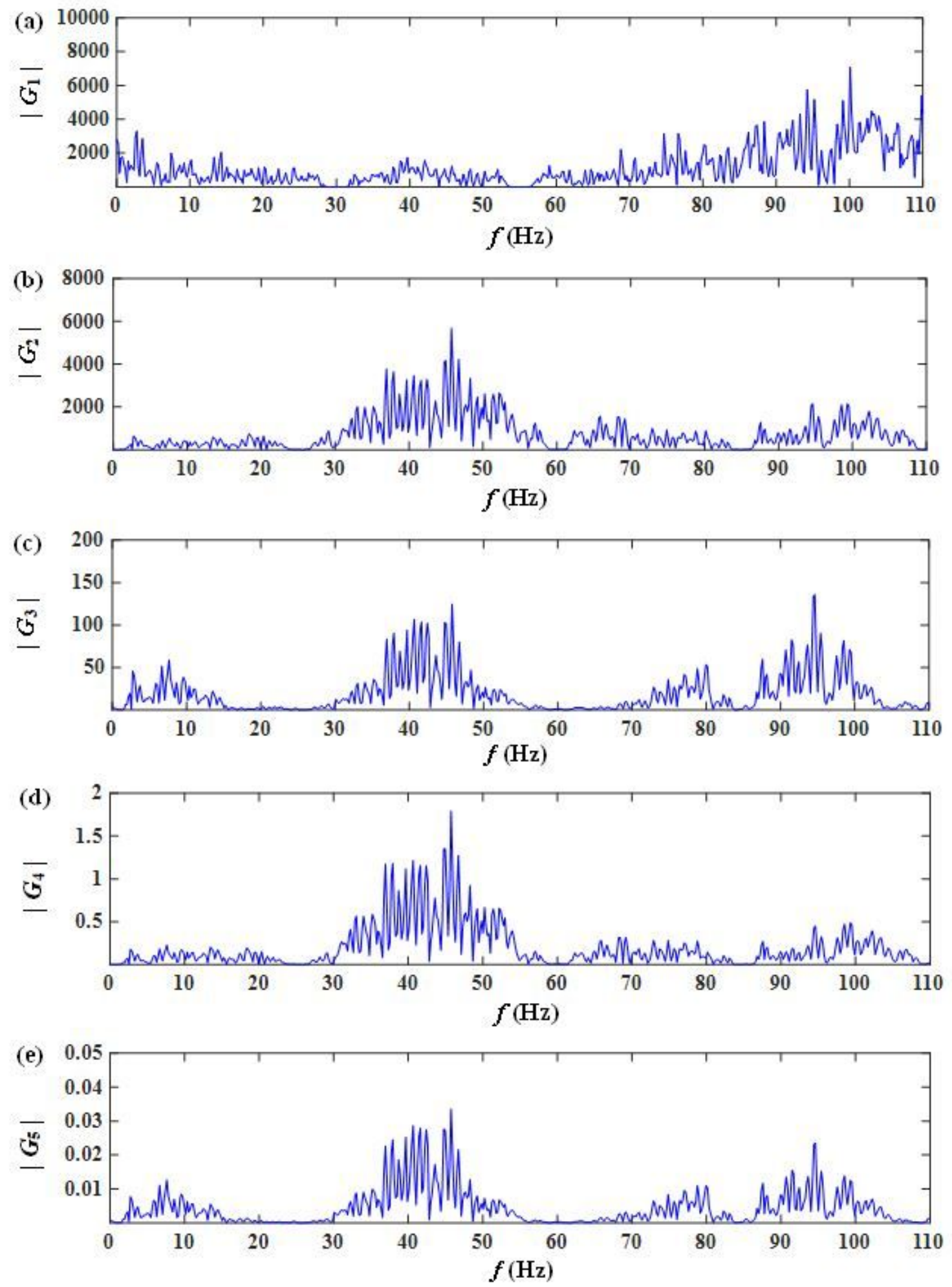

\section{Figure 15}

The amplitude spectra of the first five order NOFRFs of the second nonlinear system: (a) |G_1 (f)|; (b) |G_2 (f)|; (c) $\mid G \_3$ (f)|; (d) $\mid G_{-}$4 (f) |; (e) |G_5 (f)| 

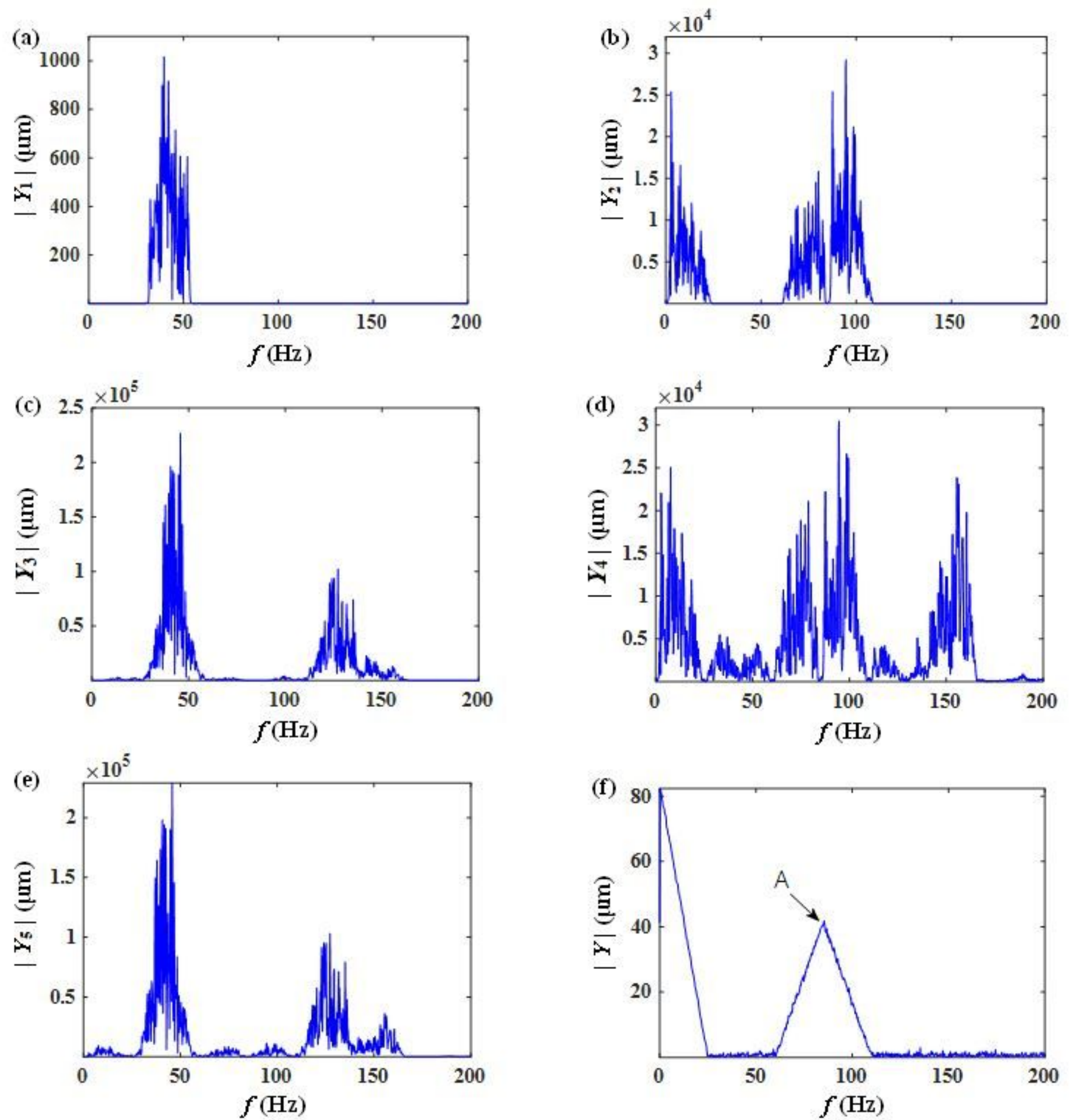

Figure 16

The amplitude spectra of the first five order unconditioned output components of the second nonlinear system and their summing spectrum: (a) |Y_1 (f)|; (b) |Y_2 (f)|; (c) |Y_3 (f)|; (d) |Y_4 (f)|; (e) |Y_5 (f)|; (f) $|\mathrm{Y}(\mathrm{f})|$ 